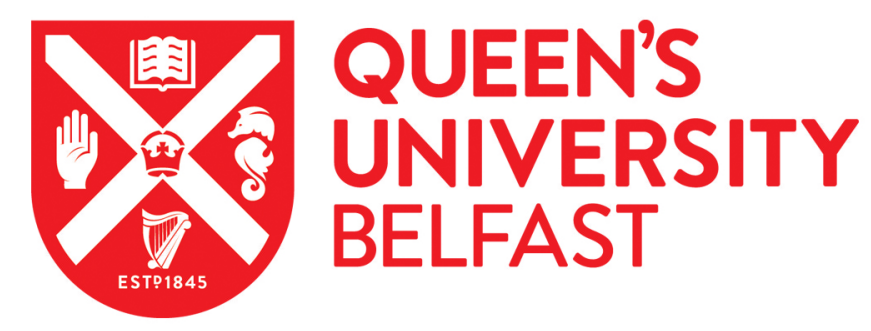

\title{
Cyclic behaviour of Volterra composition operators
}

Shkarin, S., Montes-Rodriguez, A., \& Rodriguez-Martinez, A. (2011). Cyclic behaviour of Volterra composition operators. Proceedings of the London Mathematical Society, 103(3), 535-562.

https://doi.org/10.1112/plms/pdq039

\section{Published in:}

Proceedings of the London Mathematical Society

\section{Document Version:}

Early version, also known as pre-print

\section{Queen's University Belfast - Research Portal:}

Link to publication record in Queen's University Belfast Research Portal

\section{General rights}

Copyright for the publications made accessible via the Queen's University Belfast Research Portal is retained by the author(s) and / or other copyright owners and it is a condition of accessing these publications that users recognise and abide by the legal requirements associated with these rights.

Take down policy

The Research Portal is Queen's institutional repository that provides access to Queen's research output. Every effort has been made to ensure that content in the Research Portal does not infringe any person's rights, or applicable UK laws. If you discover content in the Research Portal that you believe breaches copyright or violates any law, please contact openaccess@qub.ac.uk. 


\title{
Cyclic behaviour of Volterra composition operators
}

\author{
Alfonso Montes-Rodríguez, Alejandro Rodríguez-Martínez and Stanislav Shkarin
}

\section{Abstract}

We determine the cyclic behaviour of Volterra composition operators, which are defined as

$$
\left(V_{\varphi} f\right)(x)=\int_{0}^{\varphi(x)} f(t) d t, \quad f \in L^{p}[0,1], 1 \leqslant p \leqslant \infty,
$$

where $\varphi$ is a measurable self-map of $[0,1]$. The cyclic behaviour of $V_{\varphi}$ is essentially determined by the behaviour of the inducing symbol $\varphi$ at 0 and at 1 . As a particular result, we provide new examples of quasinilpotent supercyclic operators, which extend and complement previous ones of Héctor Salas.

\section{Introduction}

For each Lebesgue measurable self-map $\varphi$ of the unit interval $[0,1]$ and each $1 \leqslant p \leqslant \infty$, the Volterra composition operator is defined as

$$
\left(V_{\varphi} f\right)(x)=\int_{0}^{\varphi(x)} f(t) d t, \quad f \in L^{p}[0,1]
$$

which is always measurable because it is the composition of an absolutely continuous (difference of increasing functions) function with the measurable function $\varphi$. If $\varphi$ is the identity map, then the operator $V_{\varphi}$ is just the classical Volterra operator, which as usual is denoted by $V$. Observe that $V_{\varphi}=C_{\varphi} V$, where $C_{\varphi}$ denotes the operator that to each function $f$ assigns the function $f \circ \varphi$. Since $C_{\varphi}$ is bounded from $L^{\infty}[0,1]$ into itself and $V$ is bounded from $L^{p}[0,1]$ into $L^{\infty}[0,1]$ is compact (see $\left[\mathbf{1}\right.$, p. 44]), it follows that $V_{\varphi}$ acting on $L^{p}[0,1]$ is compact.

Whitley [24] and Tong [22] independently (see also [15, Corollary 2.2]) proved that $V_{\varphi}$ is quasinilpotent if and only if $\varphi(x) \leqslant x$ for each $0 \leqslant x \leqslant 1$. The cyclic behaviour of an operator depends much on the behaviour of its iterates and although there is a formula for the iterates of Volterra composition operators, this formula is not handleable. However, this handicap may be overcome.

In Section 2, we show that, for $\varphi(x)=x^{\alpha}, 0<\alpha \leqslant 1$, the operator $V_{\varphi}$ is cyclic, with cyclic vector the non-zero constant functions. Indeed, the constant function 1 is cyclic for $V_{\psi}$, with $\psi(x)=1-\varphi(1-x)$, if and only if the eigenfunctions of $V_{\varphi}$ span $L^{2}[0,1]$. In particular, there are cyclic Volterra operators with the graph of their symbols under the graph of the identity.

In Section 3, we deal with the asymptotic behaviour of the norms of iterates of $V_{\varphi}$. Indeed, for the most interesting class of symbols, the sequence $\left\{\left\|V_{\varphi}^{n}\right\|^{1 / n^{2}}\right\}$ tends to a quantity that depends only on $\varphi^{\prime}(0)$ and $\varphi^{\prime}(1)$.

Received 17 October 2008; revised 9 August 2010; published online 12 March 2011.

2000 Mathematics Subject Classification 47A10, 47B34, 31A10, 31B10.

The research was partially supported by Plan Nacional I + D + I grant no. MTM2009-09501 and Junta de Andalucía FQM-260 and P06-FQM-02225.

The results of this work are contained in the PhD Thesis of Alejandro Rodríguez-Martínez held at Universidad de Sevilla on 4 July 2007. The English version of this thesis is available at the Biblioteca de la Facultad de Matemáticas de la Universidad de Sevilla, Avda. Reina Mercedes, Sevilla 41012, Spain. 
In Section 4, in order to obtain positive results on the supercyclicity of $V_{\varphi}$ as well as on the hypercyclicity of $I+V_{\varphi}$, we need to extend Salas's theorem [19] on the hypercyclicity of perturbations of the identity by backward weighted shifts. We prove a new criterion for an operator acting on a Fréchet space to be hypercyclic.

In Section 5, we deal with supercyclicity and hypercyclicity of Volterra composition operators. Salas in [20] asked whether the classical Volterra operator is supercyclic or not, which was answered in the negative in [6] (there is another published paper by León-Saavedra and Piqueras in which they intended to prove the same result; however, their proof contains unfixable and serious errors). Indeed, the Volterra operator is not even weakly supercyclic [17]. Thus the fact that there are symbols below the main diagonal that supply supercyclic Volterra composition operators is striking. Indeed, using the results of the previous two sections, we show that, for every strictly increasing continuous $\varphi$ with $\varphi(x)<x$ for $0<x \leqslant 1$ (note that $\varphi(1)<1$ ), the operator $V_{\varphi}$ is supercyclic and the operator $I+V_{\varphi}$ is hypercyclic. For strictly increasing $\varphi$ with $\varphi(x)<x$ for $0<x<1, \varphi(1)=1$ and analytic at 0 and at 1 , it is shown that if $\varphi^{\prime}(0) \varphi^{\prime}(1)>1$, then $V_{\varphi}$ is supercyclic and if $\varphi^{\prime}(0) \varphi^{\prime}(1)<1$, then $V_{\varphi}$ is not even cyclic. The latter applies to the symbols defined by $\varphi_{\alpha}(x)=x^{\alpha}$ with $1<\alpha<\infty$. We close the section with a complete characterization of hypercyclicity of $V_{\varphi}$ on Fréchet spaces, which extends recent results by Herzog and Weber [11].

\section{Basic cyclic properties of $V_{\varphi}$ and examples}

Recall that an operator $T$ on a Banach space $\mathcal{B}$ is cyclic if there is $f$ in $\mathcal{B}$ such that $\operatorname{span}\left\{T^{n} f\right.$ : $n \geqslant 0\}$ is dense in $\mathcal{B}$. We begin with the parametric family of symbols $\varphi_{\alpha}(x)=x^{\alpha}$, which motivates a more thorough study of the symbols $\varphi$ that induce cyclic Volterra composition operators.

Proposition 2.1. Assume $\varphi(x)=x^{\alpha}$ with $\alpha>0$. Then $\tau(x)=x^{\beta}$ with $\beta>-1 / p$ is cyclic for $V_{\varphi}$ acting on $L^{p}[0,1], 1 \leqslant p<\infty$, if and only if $0<\alpha \leqslant 1$.

Proof. An elementary computation shows that $\left(V_{\varphi}^{n} \phi\right)(x)=c x^{\beta \alpha^{n}+\left(\alpha-\alpha^{n+1}\right) /(1-\alpha)}$, for each $n \geqslant 0$, where $c \neq 0$ depends on $n, \alpha$ and $\beta$ and for $\alpha=1$ the second term in the exponent does not appear. Thus, for each $1 \leqslant p<\infty$, the result follows from the Müntz theorem.

Proposition 2.1 is even true on the space $\mathcal{C}_{0}[0,1]$ of continuous functions on $[0,1]$ vanishing at 0 , endowed with the supremum norm.

The cyclicity of the constant function 1 for $V_{\varphi}$ is also possible when $\varphi(x)<x$ for $0<x<1$ (see Theorem 2.2 and Corollaries 2.3 and 2.4).

We use the notation and some results from [15]. Associated to $V_{\varphi}$ there is a function $\mathcal{F}^{\varphi}(x, z)$ defined on $[0,1] \times \mathbb{C}$. In $[\mathbf{3}]$, there is also a function $D_{V_{\varphi}}(\lambda)$ that plays the role of $\mathcal{F}^{\varphi}(0, z)$. A detailed exposition of the construction and properties of $\mathcal{F}_{x}^{\varphi}(z)=\mathcal{F}^{\varphi}(x, z)$ can be found in $[15, \S 5]$.

As usual, let $\mathcal{H}_{1 / 2}^{0}(\mathbb{C})$ denote the space of entire functions of order strictly less than $1 / 2$ or of order $1 / 2$ and type 0 . We can prove the following theorem.

Theorem 2.2. Let $\varphi$ be a continuous self-map of $[0,1]$ with $\varphi(x) \geqslant x$ for $0 \leqslant x \leqslant 1$ and set $\psi(x)=1-\varphi(1-x)$. If the span of the generalized eigenvectors of $V_{\varphi}$ is dense in $L^{2}[0,1]$, then the constant function 1 is cyclic for $V_{\psi}$. The converse is also true, provided that $\mathcal{F}_{0}^{\varphi}$ belongs to $\mathcal{H}_{1 / 2}^{0}(\mathbb{C})$. 
Proof. In [15, Proposition 5.2] it is proved that the map $x \mapsto \mathcal{F}_{x}^{\varphi}$ is continuous from $[0,1]$ into the space of entire functions. Hence, for each non-null $h$ in $L^{2}[0,1]$, we find that

$$
G^{h}(z)=\left\langle\mathcal{F}_{\varphi(\cdot)}^{\varphi}(z), h\right\rangle=\int_{0}^{1} \mathcal{F}_{\varphi(x)}^{\varphi}(z) \overline{h(x)} d x, \quad z \in \mathbb{C}
$$

is an entire function. By [15, Proposition 5.2] the Taylor coefficients of $G^{h}$ are given by

$$
G_{n}^{h}=(-1)^{n-1}\left\langle U V_{\psi}^{n} 1, h\right\rangle=(-1)^{n-1}\left\langle V_{\psi}^{n} 1, U h\right\rangle,
$$

where $(U f)(x)=f(1-x)$.

Proceeding by contradiction, suppose now that the constant function 1 is not cyclic for $V_{\psi}$. Then there is a non-zero $h$ in $L^{2}[0,1]$ such that $\left\langle V_{\psi}^{n} 1, U h\right\rangle=0$ for each $n \geqslant 0$ and, therefore, $G^{h}=0$. Thus, since in [15, Proposition 5.2] it is proved that

$$
\frac{\partial \mathcal{F}^{\varphi}}{\partial x}(x, z)=z \mathcal{F}^{\varphi}(\varphi(x), z)
$$

it follows that

$$
\int_{0}^{1} \frac{\partial \mathcal{F}^{\varphi}}{\partial x}(x, z) \overline{h(x)} d x \equiv 0 .
$$

Upon differentiating this with respect to $z$, we obtain

$$
\int_{0}^{1} \frac{\partial^{k+1} \mathcal{F}^{\varphi}}{\partial x \partial z^{k}}(x, z) \overline{h(x)} d x \equiv 0 \quad \text { for each } k \geqslant 0 .
$$

From $[\mathbf{1 5}, \S 5]$, we know that the generalized eigenfunctions of $V_{\varphi}$ belong to

$$
\operatorname{span}\left\{\frac{\partial^{k+1} \mathcal{F}^{\varphi}}{\partial x \partial z^{k}}(x, z): k=0,1, \ldots\right\} \text {. }
$$

Therefore, it follows that $h$ is orthogonal to each generalized eigenfunction of $V_{\varphi}$ and, thus, the span of the generalized eigenfunctions is not dense in $L^{2}[0,1]$, which is a contradiction.

Suppose now that $\mathcal{F}_{0}^{\varphi}$ belongs to $\mathcal{H}_{1 / 2}^{0}(\mathbb{C})$ and the constant function 1 is cyclic for $V_{\psi}$. If the span of the generalized eigenfunctions of $V_{\varphi}$ is not dense in $L^{2}[0,1]$, then there is a non-null function $h$ in $L^{2}[0,1]$ such that $h$ is orthogonal to each generalized eigenfunction of $V_{\varphi}$. Now, by the definition of $G^{h}$, the basis of (2.4) (see [15, Theorem 5.7] and (2.2)), we have that each zero of $\mathcal{F}_{0}^{\varphi}$ is also a zero of $G^{h}$ of, at least, the same multiplicity. Hence, $H(z)=G^{h}(z) / \mathcal{F}_{0}^{\varphi}(z)$ is an entire function. In addition, by [15, Corollary 5.4] and the monotonicity on $x$ of the maximum modulus of $\mathcal{F}_{x}^{\varphi}(z)$, we have that

$$
M\left(G^{h}, R\right) \leqslant \int_{0}^{1} M\left(\mathcal{F}_{\varphi(x)}^{\varphi}, R\right)|h(x)| d x \leqslant \int_{0}^{1} M\left(\mathcal{F}_{0}^{\varphi}, R\right)|h(x)| d x=M\left(\mathcal{F}_{0}^{\varphi}, R\right)\|h\|_{1} .
$$

Therefore, $G^{h}$ is in $\mathcal{H}_{1 / 2}^{0}(\mathbb{C})$, and so is $H$. Again, by [15, Corollary 5.4], we find that

$$
\left|G^{h}(-R)\right| \leqslant M\left(G^{h}, R\right) \leqslant M\left(\mathcal{F}_{0}^{\varphi}, R\right)\|h\|_{1}=\mathcal{F}_{0}^{\varphi}(-R)\|h\|_{1} .
$$

Hence, $|H(z)| \leqslant\|h\|_{1}$ for each $z$ real and negative. Since $H$ is in $\mathcal{H}_{1 / 2}^{0}(\mathbb{C})$, the PhragmenLindelöf theorem (see $\left[\mathbf{1 4}\right.$, Theorem 22, p. 50]) implies that $H$ is constant. Hence $G^{h}=c \mathcal{F}_{0}^{\varphi}$, where $c$ is a constant.

Now, for $0<x \leqslant 1$ set $\phi(x)=\inf \{t \in[0,1]: \varphi(t) \geqslant \varphi(x)\}$. Since $\varphi(x) \geqslant \phi(x)>0$ for $0<$ $x \leqslant 1$, we may apply [15, Lemma 5.9], for $\alpha=0$ and $\beta=\phi(x)$ for each $0<x \leqslant 1$, to obtain

$$
\mathcal{F}_{0}^{\varphi}(-R) \geqslant(1+\phi(x) R) \mathcal{F}_{\varphi(x)}^{\varphi}(-R) \quad \text { for each } R>0 \text { and } 0<x \leqslant 1 .
$$


This inequality, along with $c \mathcal{F}_{0}^{\varphi}=G^{h}$, implies, for each $R>0$, that

$$
\begin{aligned}
|c| \mathcal{F}_{0}^{\varphi}(-R) & \leqslant \int_{0}^{1} \mathcal{F}_{\varphi(x)}^{\varphi}(-R)|h(x)| d x \\
& \leqslant \int_{0}^{1} \frac{\mathcal{F}_{0}^{\varphi}(-R)}{1+\phi(x) R}|h(x)| d x \\
& \leqslant \mathcal{F}_{0}^{\varphi}(-R)\|h\|_{2}\left(\int_{0}^{1} \frac{d x}{(1+\phi(x) R)^{2}}\right)^{1 / 2}
\end{aligned}
$$

Therefore,

$$
\frac{|c|}{\|h\|_{L^{2}}^{2}} \leqslant \int_{0}^{1} \frac{d x}{(1+\phi(x) R)^{2}} \quad \text { for each } R>0 .
$$

Since the integral above tends to 0 as $R$ tends to $\infty$, we see that $c=0$. Thus $G^{h}$ is identically zero and so are its Taylor coefficients. Consequently, from (2.1), we find that $\left\langle V_{\psi}^{n} 1, U h\right\rangle=0$ for each $n \geqslant 0$. Since $U h$ is different from 0 , the constant function 1 cannot be cyclic for $V_{\psi}$, which is a contradiction.

As a direct consequence of [15, Corollary 5.22] and Theorem 2.2, we have the following corollary.

Corollary 2.3. Let $\varphi$ be a continuous self-map of $[0,1]$ with $\varphi(x)>x$ for $0<x<1$ and assume that

$$
\varlimsup_{x \rightarrow 0} \frac{\ln (\varphi(x)-x)}{\ln x}<2 \text { and } \varlimsup_{x \rightarrow 1} \frac{\ln (\varphi(x)-x)}{\ln (1-x)}<2 .
$$

Then the constant function 1 is cyclic for $V_{\psi}$, where $\psi(x)=1-\varphi(1-x)$, if and only if the span of the generalized eigenfunctions of $V_{\varphi}$ is dense in $L^{2}[0,1]$.

In particular, the above corollary applies to $V_{\psi}$, where $\psi(x)=1-(1-x)^{1 / 2}$. The next corollary follows from Theorem 2.2 and [15, Corollary 5.23], which ensures, under the same hypotheses on $\varphi$ in the corollary below, that the order of growth $\rho\left(\mathcal{F}_{0}^{\varphi}\right)=0$. Therefore, we have the following corollary.

Corollary 2.4. Let $\varphi$ be a continuous self-map of $[0,1]$ with $\varphi(x)>x$ for $0<x<1$. Assume also that $\varphi$ is differentiable at 0 and at 1 with $1<\varphi^{\prime}(0) \leqslant \infty$ and $\varphi^{\prime}(1)<1$. Then the constant function 1 is cyclic for $V_{\psi}$, where $\psi(x)=1-\varphi(1-x)$, if and only if the span of the eigenfunctions of $V_{\varphi}$ is dense in $L^{2}[0,1]$.

In $[\mathbf{2}, \mathbf{1 5}]$, the eigenfunctions and the eigenvalues of several parametric families of Volterra composition operators are calculated. For instance, the spectrum $\sigma\left(V_{\varphi_{\alpha}}\right)=\left\{(1-\alpha) \alpha^{n}\right\}_{n \geqslant 0} \cup$ $\{0\}$ for $\varphi_{\alpha}(x)=x^{\alpha}$ with $0<\alpha<1$. In this case, all the eigenvalues are simple and the set of eigenfunctions spans $L^{2}[0,1]$.

\section{Asymptotic behaviour of orbits of quasinilpotent $V_{\varphi}$}

If $\varphi$ is the identity map, then $V_{\varphi}=V$ for which there has been much interest on the behaviour of the norms $\left\|V^{n}\right\|_{p}, 1 \leqslant p \leqslant \infty$ (see the work by Eveson $[\mathbf{4}, \mathbf{5}]$ ), where the exact asymptotic 
behaviour of these norms is obtained using testing functions. We shall see that if $\varphi(x)<x$ for $0<x<1$, then the norms $\left\|V_{\varphi}^{n}\right\|_{p}$ tend to 0 much faster than $\left\|V^{n}\right\|_{p}$ as $n \rightarrow \infty$.

\subsection{The asymptotic behaviour of $\left\|V_{\varphi}^{n}\right\|$}

We will be mainly concerned with continuous strictly increasing symbols, since it is necessary for cyclicity of Volterra composition operators (see Section 5). However, most of the proofs in this section still work for non-increasing self-maps.

Let $\varphi$ be a continuous strictly increasing self-map of $[0,1]$ with $\varphi(x) \leqslant x$ for $0 \leqslant x \leqslant 1$ and $\varphi(1)=1$. Let $\Omega_{1}(\varphi)=[0,1]$ and, for each $n \geqslant 2$, consider

$$
\Omega_{n}(\varphi)=\left\{x \in[0,1]^{n}: x_{1} \leqslant \varphi\left(x_{2}\right), x_{2} \leqslant \varphi\left(x_{3}\right), \ldots, x_{n-1} \leqslant \varphi\left(x_{n}\right)\right\} .
$$

Let $\mu_{n}$ be the $n$-dimensional Lebesgue measure. We have the following lemma.

Lemma 3.1. Let $\varphi$ be a continuous strictly increasing self-map of $[0,1]$ with $\varphi(x) \leqslant x$ for $0 \leqslant x \leqslant 1$ and $\varphi(1)=1$. Then $\nu_{n+1}(\varphi) \leqslant\left\|V_{\varphi}^{n}\right\|_{p} \leqslant \nu_{n-1}(\varphi)$ for each $n \geqslant 2$ and $1 \leqslant p \leqslant \infty$.

Proof. Let $\mathbf{1}$ denote the function identically 1 on $[0,1]$. It is clear that $\left\|V_{\varphi}^{n} \mathbf{1}\right\|_{\infty}=$ $\left\|V_{\varphi}^{n-1} \mathbf{1}\right\|_{1}=\left(V_{\varphi}^{n} \mathbf{1}\right)(1)=\nu_{n}(\varphi)$ for $n \geqslant 1$. Hence, $\left\|V_{\varphi}^{n}\right\|_{p} \geqslant\left\|V_{\varphi}^{n} \mathbf{1}\right\|_{p} \geqslant\left\|V_{\varphi}^{n} \mathbf{1}\right\|_{1}=\nu_{n+1}(\varphi)$. We also have $\left\|V_{\varphi}^{n} f\right\|_{\infty} \leqslant\left\|V_{\varphi}^{n} \mathbf{1}\right\|_{\infty}\|f\|_{\infty}=\nu_{n}(\varphi)\|f\|_{\infty}$ for each $f \in L^{\infty}[0,1]$. Hence $\left\|V_{\varphi}^{n} f\right\|_{p} \leqslant$ $\left\|V_{\varphi}^{n-1} V_{\varphi} f\right\|_{\infty} \leqslant \nu_{n-1}(\varphi)\left\|V_{\varphi} f\right\|_{\infty} \leqslant \nu_{n-1}(\varphi)\|f\|_{p}$ for each $n \geqslant 2$. Thus, $\nu_{n+1}(\varphi) \leqslant\left\|V_{\varphi}^{n}\right\|_{p} \leqslant$ $\nu_{n-1}(\varphi)$ for any $n \geqslant 2$.

Let $\varphi$ be a continuous strictly increasing self-map of $[0,1]$ with $\varphi(x) \leqslant x$ for $0 \leqslant x \leqslant 1$. For each positive integer $n$ and each $0<a<1$, we set

$$
\Omega_{n}^{a, 0}(\varphi)=\left\{x \in \Omega_{n}(\varphi): x_{n} \leqslant a\right\} \quad \text { and } \quad \Omega_{n}^{a, 1}(\varphi)=\left\{x \in \Omega_{n}(\varphi): x_{1} \geqslant a\right\} .
$$

Lemma 3.2. Let $\varphi$ be a continuous strictly increasing self-map of $[0,1]$ with $\varphi(x)<x$ for $0<x<1$, and let $\varphi(1)=1$ and

$$
\delta_{0}^{+}=\varlimsup_{x \rightarrow 0} \frac{\varphi(x)}{x}, \quad \delta_{0}^{-}=\varliminf_{x \rightarrow 0} \frac{\varphi(x)}{x}, \quad \delta_{1}^{+}=\varlimsup_{x \rightarrow 1} \frac{1-x}{1-\varphi(x)}, \quad \delta_{1}^{-}=\varliminf_{x \rightarrow 1} \frac{1-x}{1-\varphi(x)} .
$$

Then, for each $0<a<1$, we have

$$
\begin{aligned}
& \varlimsup_{n \rightarrow \infty}\left(\mu_{n}\left(\Omega_{n}^{a, 0}(\varphi)\right)\right)^{1 / n^{2}} \leqslant \sqrt{\delta_{0}^{+}}, \quad \varliminf_{n \rightarrow \infty}\left(\mu_{n}\left(\Omega_{n}^{a, 0}(\varphi)\right)\right)^{1 / n^{2}} \geqslant \sqrt{\delta_{0}^{-}}, \\
& \varlimsup_{n \rightarrow \infty}\left(\mu_{n}\left(\Omega_{n}^{a, 1}(\varphi)\right)\right)^{1 / n^{2}} \leqslant \sqrt{\delta_{1}^{+}}, \quad \underline{\lim _{n \rightarrow \infty}}\left(\mu_{n}\left(\Omega_{n}^{a, 1}(\varphi)\right)\right)^{1 / n^{2}} \geqslant \sqrt{\delta_{1}^{-}} .
\end{aligned}
$$

In particular, if $\varphi$ is differentiable at 0 and at 1 , where $\varphi^{\prime}(1)=\infty$ is allowed, then

$$
\lim _{n \rightarrow \infty}\left(\mu_{n}\left(\Omega_{n}^{a, 0}(\varphi)\right)\right)^{1 / n^{2}}=\sqrt{\varphi^{\prime}(0)} \text { and } \lim _{n \rightarrow \infty}\left(\mu_{n}\left(\Omega_{n}^{a, 1}(\varphi)\right)\right)^{1 / n^{2}}=\sqrt{1 / \varphi^{\prime}(1)} .
$$

Proof. If $\delta_{0}^{+}=1$, then the first inequality in (3.4) becomes trivial. Indeed, if we denote by $u$ the identity function, then we have

$$
\mu_{n}\left(\Omega_{n}^{a, 0}(\varphi)\right) \leqslant \mu_{n}\left(\Omega_{n}(\varphi)\right) \leqslant \mu_{n}\left(\Omega_{n}(u)\right)=\frac{1}{(n+1) !} .
$$

Thus assume $\delta_{0}^{+}<1$. We take an arbitrary $\delta_{0}^{+}<b<1$. Clearly, there exist $0<\delta<1$ and a strictly increasing continuous self-map $\psi$ of $[0,1]$ such that $\psi(x)<x$ for $0<x<1, \psi(x)=b x$ for $0 \leqslant x \leqslant \delta$ and $\psi(x) \geqslant \varphi(x)$ for $0 \leqslant x \leqslant 1$. Since $\psi(x)<x$ for $0<x<1$, we find that there 
is a positive integer $k$ such that $\psi_{k}(a) \leqslant \delta$, where $\psi_{k}$ is the $k$ th iterate of $\psi$. It immediately follows that $\mu_{n}\left(\Omega_{n}^{a, 0}(\psi)\right) \leqslant \mu_{n-k}\left(\Omega_{n-k}^{\delta, 0}(\psi)\right)$ for $n>k$. Since $\psi(x)=b x$ for $0 \leqslant x \leqslant \delta$, we have

$$
\mu_{j}\left(\Omega_{j}^{\delta, 0}(\psi)\right)=\int_{0}^{\delta} d x_{j} \int_{0}^{b x_{j}} d x_{j-1} \cdots \int_{0}^{b x_{3}} d x_{2} \int_{0}^{b x_{2}} d x_{1}=\frac{\delta^{j} b^{j(j-1) / 2}}{j !}
$$

for each $j \geqslant 1$. Since $\mu_{n}\left(\Omega_{n}^{a, 0}(\varphi)\right) \leqslant \mu_{n}\left(\Omega_{n}^{a, 0}(\psi)\right)$ for each $n \geqslant 1$, from (3.8) it follows that $\varlimsup_{n \rightarrow \infty}\left(\mu_{n}\left(\Omega_{n}^{a, 0}(\varphi)\right)\right)^{1 / n^{2}} \leqslant \sqrt{b}$. Since $\delta_{0}^{+}<b<1$ was arbitrary, the first inequality in (3.4) follows.

If $\delta_{0}^{-}=0$, then the second inequality in (3.4) is trivial. Thus assume $\delta_{0}^{-}>0$. We take an arbitrary $0<b<\delta_{0}^{-}$. Clearly, there is $0<\delta<a$ and a strictly increasing continuous self-map $\psi$ of $[0,1]$ such that $\psi(x)<x$ for $0<x<1, \psi(x)=b x$ for $0 \leqslant x \leqslant \delta$ and $\psi(x) \leqslant \varphi(x)$ for $0 \leqslant x \leqslant 1$. Since $\mu_{n}\left(\Omega_{n}^{a, 0}(\varphi)\right) \geqslant \mu_{n}\left(\Omega_{n}^{a, 0}(\psi)\right) \geqslant \mu_{n}\left(\Omega_{n}^{\delta, 0}(\psi)\right)$ for each $n \geqslant 1$, from (3.8) we obtain

$$
\varliminf_{n \rightarrow \infty}\left(\mu_{n}\left(\Omega_{n}^{a, 0}(\varphi)\right)\right)^{1 / n^{2}} \geqslant \sqrt{b} .
$$

Since $0<b<\delta_{0}^{-}$was arbitrary, the second inequality in (3.4) also follows.

Finally, since $\varphi$ satisfies (3.5) if and only if $\phi(x)=1-\varphi^{-1}(1-x)$ satisfies (3.4), the proof of the statement of the lemma is complete.

In order to state the main result of this section, we consider

$$
\phi(u, v)= \begin{cases}\exp \left(\frac{\ln u \ln v}{2 \ln (u v)}\right) & \text { if } u>0, v>0 \text { and }(u, v) \neq(1,1) \\ \sqrt{|u-v|} & \text { if } u=0 \text { or } v=0 \\ 1 & \text { if }(u, v)=(1,1)\end{cases}
$$

which is clearly continuous on $[0,1]^{2}$ and takes its values in $[0,1]$.

THEOREM 3.3. Let $\varphi$ be a continuous strictly increasing self-map of $[0,1]$ with $\varphi(x)<x$ for $0<x<1$, and let $\varphi(1)=1$ and $\delta_{0}^{+}, \delta_{0}^{-}, \delta_{1}^{+}, \delta_{1}^{-}$be as in (3.3). Then, for $1 \leqslant p \leqslant \infty$, we have

$$
\rho_{-} \leqslant \varliminf_{n \rightarrow \infty}\left\|V_{\varphi}^{n}\right\|_{p}^{1 / n^{2}} \leqslant \varlimsup_{n \rightarrow \infty}\left\|V_{\varphi}^{n}\right\|_{p}^{1 / n^{2}} \leqslant \rho_{+},
$$

where $\rho_{-}=\phi\left(\delta_{0}^{-}, \delta_{1}^{-}\right)$and $\rho_{+}=\phi\left(\delta_{0}^{+}, \delta_{1}^{+}\right)$. In particular, if $\varphi$ is differentiable at 0 and at 1 , then

$$
\lim _{n \rightarrow \infty}\left\|V_{\varphi}^{n}\right\|^{1 / n^{2}}=\phi\left(\varphi^{\prime}(0), 1 / \varphi^{\prime}(1)\right) .
$$

Proof. According to Lemma 3.1, it is enough to show that

$$
\rho_{-} \leqslant \varliminf_{n \rightarrow \infty}\left(\nu_{n}(\varphi)\right)^{1 / n^{2}} \leqslant \varlimsup_{n \rightarrow \infty}\left(\nu_{n}(\varphi)\right)^{1 / n^{2}} \leqslant \rho_{+} .
$$

If $\rho_{+}=1$, then the last inequality in (3.9) follows from the second one in (3.7). Thus assume that $\rho_{+}<1$. Hence, we must have $\delta_{0}^{+}<1$ and $\delta_{1}^{+}<1$. We take $\delta_{0}^{+}<b_{0}<1$ and $\delta_{1}^{+}<b_{1}<1$. By Lemma 3.2, there is $c>0$ such that

$$
\mu_{n}\left(\Omega_{n}^{1 / 2,0}(\varphi)\right) \leqslant c b_{0}^{n^{2} / 2} \text { and } \mu_{n}\left(\Omega_{n}^{1 / 2,1}(\varphi)\right) \leqslant c b_{1}^{n^{2} / 2}
$$

for each positive integer $n$. Clearly, $\Omega_{n}(\varphi) \subset \bigcup_{k=0}^{n} A_{k}$, where $A_{0}=\Omega_{n}^{1 / 2,0}(\varphi), A_{n}=\Omega_{n}^{1 / 2,1}(\varphi)$ and $A_{k}=\Omega_{n-k}^{1 / 2,0}(\varphi) \times \Omega_{k}^{1 / 2,1}(\varphi)$ for $0<k<n$. Hence,

$$
\nu_{n}(\varphi) \leqslant \sum_{k=0}^{n} \mu_{n}\left(A_{k}\right)=\sum_{k=0}^{n} \mu_{n-k}\left(\Omega_{n-k}^{1 / 2,0}(\varphi)\right) \mu_{k}\left(\Omega_{k}^{1 / 2,1}(\varphi)\right) .
$$


Using (3.10), we obtain

$$
\begin{aligned}
\nu_{n}(\varphi) & \leqslant c^{2} \sum_{k=0}^{n} b_{0}^{(n-k)^{2} / 2} b_{1}^{k^{2} / 2} \\
& \leqslant c^{2}(n+1) \max _{0 \leqslant k \leqslant n} b_{0}^{(n-k)^{2} / 2} b_{1}^{k^{2} / 2} \\
& \leqslant c^{2}(n+1)\left(\max _{[0,1]} b_{0}^{(1-x)^{2} / 2} b_{1}^{x^{2} / 2}\right)^{n^{2}} .
\end{aligned}
$$

The last maximum is attained for $x=\ln b_{0} / \ln \left(b_{0} b_{1}\right)$ and equals $\phi\left(b_{0}, b_{1}\right)$. Therefore, $\varlimsup_{n \rightarrow \infty}\left(\nu_{n}(\varphi)\right)^{1 / n^{2}} \leqslant \phi\left(b_{1}, b_{2}\right)$. Since $\delta_{0}^{+}<b_{0}<1$ and $\delta_{1}^{+}<b_{1}<1$ were arbitrary, the last inequality of (3.9) is satisfied.

If $\rho_{-}=0$, then the first inequality in (3.9) is trivial. Thus assume $\rho_{-}>0$. Hence, we must have $\delta_{0}^{-}>0$ and $\delta_{1}^{-}>0$. We take $0<b_{0}<\delta_{0}^{-}$and $0<b_{1}<\delta_{1}^{-}$. Let $a>0$ be small enough to ensure that $a<\varphi(1-a)$. By Lemma 3.2, there is $c>0$ such that

$$
\mu_{n}\left(\Omega_{n}^{a, 0}(\varphi)\right) \geqslant c b_{0}^{n^{2} / 2} \text { and } \mu_{n}\left(\Omega_{n}^{1-a, 1}(\varphi)\right) \geqslant c b_{1}^{n^{2} / 2}
$$

for each $n \geqslant 1$. Choose a sequence $\left\{k_{n}\right\}_{n \geqslant 1}$ of positive integers such that $k_{n}<n$ for each $n$ and $k_{n} / n$ tends to $\ln b_{1} / \ln \left(b_{1} b_{2}\right)$ as $n$ tends to $\infty$. Clearly, $\Omega_{n}(\varphi) \supset A=\Omega_{n-k_{n}}^{a, 0}(\varphi) \times \Omega_{k_{n}}^{1-a, 1}(\varphi)$. Hence, $\nu_{n}(\varphi) \geqslant \mu_{n}(A)=\mu_{n-k_{n}}\left(\Omega_{n-k_{n}}^{a, 0}(\varphi)\right) \mu_{k_{n}}\left(\Omega_{k_{n}}^{1-a, 1}(\varphi)\right)$. Using (3.11), we obtain

$$
\nu_{n}(\varphi) \geqslant c^{2} b_{0}^{\left(n-k_{n}\right)^{2} / 2} b_{1}^{k_{n}^{2} / 2}=c^{2}\left(b_{0}^{\left(1-\left(k_{n} / n\right)\right)^{2}} b_{1}^{\left(k_{n} / n\right)^{2}}\right)^{n^{2} / 2} .
$$

Since $k_{n} / n$ tends to $\ln b_{0} / \ln \left(b_{0} b_{1}\right)$, we see that $\lim _{n \rightarrow \infty} b_{0}^{1-k_{n} / n} b_{1}^{k_{n} / n}=\phi\left(b_{0}, b_{1}\right)$. Thus we obtain $\underline{\lim }_{n \rightarrow \infty}\left(\nu_{n}(\varphi)\right)^{1 / n^{2}} \geqslant \phi\left(b_{0}, b_{1}\right)$. Since $0<b_{0}<\delta_{0}^{-}$and $0<b_{1}<\delta_{1}^{-}$were arbitrary, the first inequality in (3.9) also holds. The proof is complete.

Corollary 3.4. Let $\varphi$ be a continuous strictly increasing self-map of $[0,1]$ with $\varphi(x)<x$ for $0<x<1$, and let $\varphi(1)=1$ and $\varphi$ be differentiable at 0 and 1 . If $\varphi^{\prime}(0)=0$, then $\lim _{n \rightarrow \infty}\left\|V_{\varphi}^{n}\right\|_{p}^{1 / n^{2}}=1 / \sqrt{\varphi^{\prime}(1)}$ and if $\varphi^{\prime}(1)=\infty$, then $\lim _{n \rightarrow \infty}\left\|V_{\varphi}^{n}\right\|_{p}^{1 / n^{2}}=\sqrt{\varphi^{\prime}(0)}$ for $1 \leqslant p \leqslant \infty$.

\subsection{Orbits of $V_{\varphi}$ : upper estimate}

The next lemma will be very useful to determine the cyclic properties of $V_{\varphi}$.

Lemma 3.5. Let $\varphi$ be a continuous strictly increasing self-map of $[0,1]$ with $\varphi(x)<x$ for $0<x<1$, and let $\varphi(1)=1$ and

$$
\delta_{1}^{+}=\delta_{1}^{+}(\varphi)=\varlimsup_{x \rightarrow 1} \frac{1-x}{1-\varphi(x)} .
$$

Assume also that $f$ in $L^{p}[0,1], 1 \leqslant p \leqslant \infty$, satisfies $\inf \operatorname{supp}(f)>0$. Then, $\overline{\lim }_{n \rightarrow \infty}\left\|V_{\varphi}^{n} f\right\|_{p}^{1 / n^{2}} \leqslant$ $\sqrt{\delta_{1}^{+}}$. In particular, for $\varphi$ differentiable at 1 , we have that $\overline{\lim }_{n \rightarrow \infty}\left\|V_{\varphi}^{n} f\right\|_{p}^{1 / n^{2}} \leqslant \sqrt{1 / \varphi^{\prime}(1)}$.

Proof. Let $\varepsilon>0$ be such that $f$ vanishes on $[0, \varepsilon]$. Since $V_{\varphi} f$ is continuous and also vanishes $[0, \varepsilon]$, there is $c>0$ for which $\left|\left(V_{\varphi} f\right)(x)\right| \leqslant c \chi_{[\varepsilon, 1]}(x)$ for each $0 \leqslant x \leqslant 1$, where $\chi_{[\varepsilon, 1]}$ is the characteristic function of $[\varepsilon, 1]$. Hence,

$$
\left\|V_{\varphi}^{n} f\right\|_{p} \leqslant\left\|V_{\varphi}^{n} f\right\|_{\infty} \leqslant c\left\|V_{\varphi}^{n} \chi_{[\varepsilon, 1]}\right\|_{\infty}=c\left(V_{\varphi}^{n} \chi_{[\varepsilon, 1]}\right)(1) .
$$


Let $\Omega_{n}^{\varepsilon, 1}(\varphi)$ be as in (3.2). Then $\left(V_{\varphi}^{n} \chi_{[\varepsilon, 1]}\right)(1)=\mu_{n}\left(\Omega_{n}^{\varepsilon, 1}(\varphi)\right)$ for each positive integer $n$. Therefore, by Lemma 3.2, we have

$$
\varlimsup_{n \rightarrow \infty}\left\|V_{\varphi}^{n} \chi_{[\varepsilon, 1]}\right\|_{1}^{1 / n^{2}}=\varlimsup_{n \rightarrow \infty}\left(\mu_{n}\left(\Omega_{n}^{\varepsilon, 1}(\varphi)\right)\right)^{1 / n^{2}} \leqslant \sqrt{\delta_{1}^{+}} .
$$

The required result follows immediately from the previous inequalities.

\subsection{The backward orbits of $V_{\varphi}$}

In this subsection, we consider the asymptotic behaviour of certain backward orbits of $V_{\varphi}$. In the next two sections, we shall apply these results to determine the cyclic behaviour of Volterra composition operators.

If $S$ is any linear operator acting on a linear space $X$, then $S^{\infty}(X)=\bigcap_{n=0}^{\infty} S^{n}(X)$ is a subspace of $X$ invariant under $S$. Moreover, since $S\left(S^{\infty}(X)\right)=S^{\infty}(X)$, the restriction of $S$ to $S^{\infty}(X)$ is always onto. In addition, if $\operatorname{ker} S=\{0\}$, then $S$ is one-to-one from $S^{\infty}(X)$ onto itself. Thus the backward orbits of any $x$ in $S^{\infty}(X)$ are well defined. This is in particular our case when $\operatorname{ker} V_{\varphi}=\{0\}$.

Recall that $\mathcal{C}_{0}[0,1]$ is the subspace of $\mathcal{C}[0,1]$ of functions vanishing at 0 , endowed with the supremum norm.

THEOREM 3.6. Let $\varphi$ be a continuous strictly increasing self-map of $[0,1]$ with $\varphi(x)<x$ for $0<x<1$ and $\varphi(1)=1$. Assume also that $\varphi$ is analytic at 0 and $\varphi^{\prime}(0)>0$. Then, for each $b>1 / \varphi^{\prime}(0)$, the set $F_{b}=\left\{f \in V_{\varphi}^{\infty}\left(\mathcal{C}_{0}[0,1]\right)\right.$ such that $\left.\varlimsup_{n \rightarrow \infty}\left\|V_{\varphi}^{-n} f\right\|_{\infty}^{1 / n^{2}} \leqslant \sqrt{b}\right\}$ is a dense linear manifold of $\mathcal{C}_{0}[0,1]$ satisfying $V_{\varphi}\left(F_{b}\right)=V_{\varphi}^{-1}\left(F_{b}\right)=F_{b}$.

The remainder of this section is devoted to showing Theorem 3.6. In order to do so, we need the following well-known criterion of analyticity. For each $f \in \mathcal{C}^{\infty}[a, b]$ we set

$$
M_{n}(f)=\frac{1}{n !} \max _{[a, b]}\left|f^{(n)}\right| .
$$

Then $f$ is analytic on $[u, v]$ if and only if

$$
\varlimsup_{n \rightarrow \infty}\left(M_{n}(f)\right)^{1 / n}<\infty .
$$

We also need the space $\mathcal{F}[a, b]$ defined as

$$
\left\{f \in \mathcal{C}^{\infty}[a, b]: f^{(n)}(a)=f^{(n)}(b)=0 \text { for each } n \geqslant 0 \text { and } \varlimsup_{n \rightarrow \infty}\left(M_{n}(f)\right)^{1 / n^{2}} \leqslant 1\right\} .
$$

By means of Leibniz's formula for the derivatives of a product, it is easy to check that if $f$ is in $\mathcal{F}[a, b]$ and $g$ in $\mathcal{C}^{\infty}[a, b]$ satisfies $\varlimsup_{n \rightarrow \infty}\left(M_{n}(g)\right)^{1 / n^{2}} \leqslant 1$, then $f g$ belongs to $\mathcal{F}[a, b]$. In particular, the space $\mathcal{F}[a, b]$ is an algebra with respect to pointwise multiplication and is invariant under multiplication by analytic functions.

Lemma 3.7. Assume that $-\infty<a<b<\infty$. Then

$$
h(x)= \begin{cases}e^{-1 /(x-a)-1 /(b-x)} & \text { if } a<x<b, \\ 0 & \text { if } x=a \text { or } x=b\end{cases}
$$

belongs to $\mathcal{F}[a, b]$. 
Proof. Clearly, $h$ is in $\mathcal{C}^{\infty}[a, b]$ with $h^{(n)}(a)=h^{(n)}(b)=0$ for each $n \geqslant 0$. Thus we need only prove that

$$
\varlimsup_{n \rightarrow \infty}\left(M_{n}(h)\right)^{1 / n^{2}} \leqslant 1 .
$$

To this end, we estimate $M_{n}(g)$, where $g(x)=e^{-1 / x}$ for $x>0$ and $g(0)=0$. By induction, one easily sees that $g^{(n)}(x)=p_{n}(1 / x) g(x)$ for $x \neq 0$, where

$$
p_{0}=1 \quad \text { and } p_{n+1}(t)=t^{2} p_{n}(t)-t^{2} p_{n}^{\prime}(t) \text { for each } n \geqslant 1 \text {. }
$$

Clearly, $p_{n}(t)=\sum_{j=0}^{2 n} a_{n, j} t^{j}$, where the coefficients $a_{n, j}$ are real. Therefore,

$$
M_{n}(g)=\frac{1}{n !} \sup _{x>0}\left|g^{(n)}(x)\right| \leqslant \frac{1}{n !} \sum_{j=0}^{2 n}\left|a_{n, j}\right| \sup _{x>0} x^{-j} e^{-1 / x}=\frac{1}{n !} \sum_{j=0}^{2 n}\left|a_{n, j}\right| \sup _{x>0} x^{j} e^{-x} .
$$

Since $\sup _{x>0} x^{j} e^{-x}=(j / e)^{j}$, we have $n ! M_{n}(g) \leqslant \sum_{j=0}^{2 n}\left|a_{n, j}\right|(j / e)^{j} \leqslant(2 n / e)^{2 n} \sigma_{n}$, where $\sigma_{n}=$ $\sum_{j=0}^{2 n}\left|a_{n, j}\right|$. Using (3.16), we see that $\sigma_{n+1} \leqslant(2 n+1) \sigma_{n}$ and, therefore, $\sigma_{n} \leqslant(2 n) ! /\left(2^{n} n !\right)$. Upon putting everything together and using Stirling's formula, one easily sees that $M_{n}(g) \leqslant$ $(2 n / e)^{2 n}$.

Since $h(x)=g(x-a) g(b-x)$, applying Leibniz's formula, we see that

$$
h^{(n)}(x)=\sum_{k=0}^{n} \frac{(-1)^{k} n !}{k !(n-k) !} g^{(k)}(b-x) g^{(n-k)}(x-a) \quad \text { for } a \leqslant x \leqslant b .
$$

Therefore, using that $M_{k}(g) \leqslant(2 k / e)^{2 k}$ in the second inequality below, we have

$$
M_{n}(h) \leqslant \sum_{k=0}^{n} \frac{n !}{k !(n-k) !} M_{k}(g) M_{n-k}(g) \leqslant 2^{n} \max _{0 \leqslant k \leqslant n}(2 k)^{2 k}(2 n-2 k)^{2 n-2 k}<(2 n)^{2 n},
$$

from which (3.15) follows and the result is proved.

The following lemma can also be derived from the Denjoy-Carleman theorem (see [18, p. 380]). Here, we provide an easy elementary proof. We denote by $\mathcal{C}_{00}[a, b]$ the Banach subspace of $\mathcal{C}[a, b]$ that consists of functions that vanish at $a$ and $b$.

Lemma 3.8. Assume that $a<b$ are real. Then $\mathcal{F}[a, b]$ is dense in $\mathcal{C}_{00}[a, b]$ and $\mathcal{F}^{+}[a, b]=\{f \in \mathcal{F}[a, b]$ such that $f(x) \geqslant 0$ for each $x \in[a, b]\}$ is dense in $\mathcal{C}_{00}^{+}[a, b]=\{f \in$ $\mathcal{C}_{00}[a, b]$ such that $f(x) \geqslant 0$ for each $\left.x \in[a, b]\right\}$.

Proof. Let $h$ be the function in (3.14). By Lemma 3.7, we know that $h$ is in $\mathcal{F}[a, b]$. Since $h$ is in $\mathcal{C}_{00}[a, b]$ and $h(x)>0$ for $a<x<b$, we see that the set $W$ consisting of functions $p h$ such that $p$ is a polynomial is dense in $\mathcal{C}_{00}[a, b]$. Also, the set $W^{+}$consisting of functions $g \in W$ such that $g(x) \geqslant 0$ on $[a, b]$ is dense in $\mathcal{C}_{00}^{+}[a, b]$. Now, the result follows because $\mathcal{F}[a, b]$ is stable with respect to multiplication by polynomials and therefore $W \subset \mathcal{F}[a, b]$ and $W^{+} \subset \mathcal{F}^{+}[a, b]$.

We need an operator related to the local inverse of $V_{\varphi}$. For $0<a<1$, we set

$$
\mathcal{E}_{a}=\left\{f \in \mathcal{C}^{\infty}[0,1] \text { such that supp } f \subset[0, a] \text { and } f^{(n)}(0)=0 \text { for } n \geqslant 0\right\} .
$$

For an analytic function $\psi:[0, a] \rightarrow[0,1]$ such that $\psi(a) \geqslant a$ and $\psi(0)=0$, consider the operator $T_{\psi}: \mathcal{E}_{a} \rightarrow \mathcal{E}_{a}$ defined as

$$
\left(T_{\psi} f\right)(x)= \begin{cases}f^{\prime}(\psi(x)) & \text { if } x \in[0, a] \\ 0 & \text { if } x \in(a, 1]\end{cases}
$$


The requirements $\psi(a) \geqslant a$ and $\psi(0)=0$ imply that $T_{\psi}$ acts from $\mathcal{E}_{a}$ into itself. As usual, for each pair $n$ and $l$ of non-negative integers, we write $(n)_{l}=1$ for $l=0$ and $(n)_{l}=$ $(n+1) \ldots(n+l)$ for $l>0$.

To prove Lemmas 3.10, 3.13 and 3.15, we need the Faá di Bruno formula for the $n$th derivative of a type (see [15, Lemma 6.1] or [21, Chapter 3]).

Lemma 3.9. Let $f$ and $g$ be in $\mathcal{C}^{n}[u, v]$. Then, for each $u \leqslant x \leqslant v$, we have

$$
(g \circ f)^{(n)}(x)=n ! \sum_{k_{1}+\ldots+n k_{n}=n} \frac{g^{\left(k_{1}+\ldots+k_{n}\right)}(f(x))}{k_{1} ! \ldots k_{n} !(1 !)^{k_{1}} \ldots(n !)^{k_{n}}}\left(f^{\prime}(x)\right)^{k_{1}} \ldots\left(f^{(n)}(x)\right)^{k_{n}} .
$$

Here $k_{1}+\ldots+n k_{n}$ indicates that the sum runs through all the $n$-tuples such that $\sum_{j=1}^{n} j k_{j}=n$. An immediate consequence of the above lemma (see, for instance, $[\mathbf{1 5}$, Section 6$]$ ) is that, for each $c \in \mathbb{C}$ and each $n \geqslant 1$, the following holds:

$$
\sum_{k_{1}+\ldots+n k_{n}=n} \frac{\left(k_{1}+\ldots+k_{n}\right) !}{k_{1} ! \ldots k_{n} !} c^{k_{1}+\ldots+k_{n}}=c(c+1)^{n-1} .
$$

Lemma 3.10. Let $\psi$ be an analytic function from $[0, a]$ into $[0,1]$, where $0<a<1$, with $\psi(0)=0$ and $\psi(a) \geqslant a$. Let $\left\{c_{n}\right\}_{n \geqslant 0}$ be such that $c_{n} \geqslant 1$ with $\lim _{n \rightarrow \infty} c_{n}^{1 / n}=1$ and let $\left\{f_{n}\right\}_{n \geqslant 0}$ be in $\mathcal{E}_{a}$ satisfying $\beta_{n}=\sup _{k \geqslant 0} M_{n}\left(f_{k}\right) c_{k}^{-n-1}<\infty$, for each $n \geqslant 0$ and $\overline{\lim }_{n \rightarrow \infty} \beta_{n}^{1 / n^{2}} \leqslant 1$. Then $\overline{\lim }_{n \rightarrow \infty}\left\|T_{\psi}^{n} f_{n}\right\|_{\infty}^{1 / n^{2}} \leqslant \sqrt{\gamma}$.

Proof. The proof is split into three steps.

Step 1. Let $\left\{\widehat{\beta}_{n}\right\}_{n \geqslant 0}$ be a sequence such that $\left\{\widehat{\beta}_{n}^{1 / n}\right\}$ is increasing. Assume also that $c>0$ and $l$ is a non-negative integer. Then, for $f$ in $\mathcal{E}_{a}$ satisfying $M_{n}(f) \leqslant c(n)_{l} \widehat{\beta}_{n}$ for each $n \geqslant 0$, we have

$$
M_{n}\left(T_{\psi} f\right) \leqslant c(n)_{l+1} \gamma^{n} \widehat{\beta}_{n+1}\left(1+\frac{R}{\gamma \widehat{\beta}_{n+1}^{1 /(n+1)}}\right)^{n} \quad \text { for each } n \geqslant 0,
$$

where

$$
R=\sup _{n \geqslant 2}\left(\frac{M_{n}(\psi)}{\gamma}\right)^{1 /(n-1)}
$$

Proof of Step 1. Since $\psi$ is analytic on $[0, a]$, by (3.13), we see that $R$ is finite. Clearly, $\left\|T_{\psi} f\right\|_{\infty} \leqslant\left\|f^{\prime}\right\|_{\infty}=M_{1}(f) \leqslant c(1)_{l} \widehat{\beta}_{1}=c(0)_{l+1} \widehat{\beta}_{1}$. Thus the result is true for $n=0$. Since

$$
M_{n}(\psi) \leqslant \gamma R^{n-1} \text { for } n \geqslant 1,
$$

using Lemma 3.9, for each $0 \leqslant x \leqslant a$ with $0<\psi(x)<a$ and for each $n \geqslant 1$, we have

$$
\left(T_{\psi} f\right)^{(n)}(x)=\left(f^{\prime} \circ \psi\right)^{(n)}(x)=n ! \sum_{n=k_{1}+\ldots+n k_{n}} \frac{f^{\left(k_{1}+\ldots+k_{n}+1\right)}(\psi(x))}{k_{1} ! \ldots k_{n} !(1 !)^{k_{1}} \ldots(n !)^{k_{n}}}\left(\psi^{\prime}(x)\right)^{k_{1}} \ldots\left(\psi^{(n)}(x)\right)^{k_{n}} .
$$

From (3.18), we have $\left(T_{\psi} f\right)^{(n)}(x)=0$ for $f$ in $\mathcal{E}_{a}$ and $\psi(x) \geqslant a$ and, therefore, we may write

$$
\begin{aligned}
M_{n}\left(T_{\psi} f\right) & \leqslant \sum_{n=k_{1}+\ldots+n k_{n}} \frac{\left(k_{1}+\ldots+k_{n}+1\right) !}{k_{1} ! \ldots k_{n} !} M_{k_{1}+\ldots+k_{n}+1}(f)\left(M_{1}(\psi)\right)^{k_{1}} \ldots\left(M_{n}(\psi)\right)^{k_{n}} \\
& \leqslant(n+1) \sum_{n=k_{1}+\ldots+n k_{n}} \frac{\left(k_{1}+\ldots+k_{n}\right) !}{k_{1} ! \ldots k_{n} !} M_{k_{1}+\ldots+k_{n}+1}(f)\left(M_{1}(\psi)\right)^{k_{1}} \ldots\left(M_{n}(\psi)\right)^{k_{n}} .
\end{aligned}
$$


From (3.22) and the fact that $M_{k}(f) \leqslant c(k)_{l} \widehat{\beta}_{k}$, we have

$$
M_{n}\left(T_{\psi} f\right) \leqslant c(n)_{l+1} \sum_{n=k_{1}+\ldots+n k_{n}} \frac{\left(k_{1}+\ldots+k_{n}\right) !}{k_{1} ! \ldots k_{n} !} \widehat{\beta}_{k_{1}+\ldots+k_{n}+1} \gamma^{k_{1}}(\gamma R)^{k_{2}} \ldots\left(\gamma R^{n-1}\right)^{k_{n}} .
$$

Since $\left\{\widehat{\beta}_{k}^{1 / k}\right\}$ is increasing, it follows that $\widehat{\beta}_{k} \leqslant\left(\widehat{\beta}_{m}\right)^{k / m}$ for $1 \leqslant k \leqslant m$. Therefore,

$$
M_{n}\left(T_{\psi} f\right) \leqslant c(n)_{l+1} R^{n} \widehat{\beta}_{n+1}^{1 /(n+1)} \sum_{n=k_{1}+\ldots+n k_{n}} \frac{\left(k_{1}+\ldots+k_{n}\right) !}{k_{1} ! \ldots k_{n} !}\left(\frac{\gamma \widehat{\beta}_{n+1}^{1 /(n+1)}}{R}\right)^{k_{1}+\ldots+k_{n}} .
$$

Applying (3.20), we have

$$
\begin{aligned}
M_{n}\left(T_{\psi} f\right) & \leqslant c(n)_{l+1} \gamma R^{n-1} \widehat{\beta}_{n+1}^{2 /(n+1)}\left(1+\frac{\gamma \widehat{\beta}_{n+1}^{1 /(n+1)}}{R}\right)^{n-1} \\
& =c(n)_{l+1} \gamma^{n} \widehat{\beta}_{n+1}\left(1+\frac{R}{\gamma \widehat{\beta}_{n}^{1 /(n+1)}}\right)^{n-1} \\
& \leqslant c(n)_{l+1} \gamma^{n} \widehat{\beta}_{n+1}\left(1+\frac{R}{\gamma \widehat{\beta}_{n}^{1 /(n+1)}}\right)^{n} .
\end{aligned}
$$

The proof of Step 1 is complete.

Step 2. Under the hypotheses of the lemma, we have that $\widetilde{\beta}_{n}=\sup _{k \geqslant 0} M_{n}\left(T_{\psi} f_{k}\right) c_{k}^{-n-2}$ is finite for each $n \geqslant 0$ and $\overline{\lim }_{n \rightarrow \infty} \widetilde{\beta}_{n}^{1 / n^{2}} \leqslant 1$.

Proof of Step 2. Let $\delta>1$ be fixed. Since $\varlimsup_{n \rightarrow \infty} \beta_{n}^{1 / n^{2}} \leqslant 1$, we may choose $C>0$ such that $\beta_{n} \leqslant C \delta^{n^{2}}$ for each $n \geqslant 0$. For each $n \geqslant 0$, we set $\widehat{\beta}_{n}=c_{k}^{n} \delta^{n^{2}}$, where $c=C c_{k}$. Then, for each $k \geqslant 0$, we have $M_{n}\left(f_{k}\right) \leqslant c \widehat{\beta}_{n}$. By Step 1 , we have

$$
M_{n}\left(T_{\psi} f_{k}\right) \leqslant c(n+1) \gamma^{n} \widehat{\beta}_{n+1}\left(1+\frac{R}{\gamma \widehat{\beta}_{n+1}^{1 /(n+1)}}\right)^{n} \quad \text { for each } n \geqslant 0 .
$$

Upon substituting the values of $c$ and $\widehat{\beta}_{n+1}$, we obtain

$$
\begin{aligned}
M_{n}\left(T_{\psi} f_{k}\right) & \leqslant C(n+1) c_{k}^{n+2} \gamma^{n} \delta^{(n+1)^{2}}\left(1+\frac{R}{\gamma c_{k} \delta^{n+1}}\right)^{n} \\
& \leqslant C(n+1) c_{k}^{n+2} \gamma^{n} \delta^{(n+1)^{2}}\left(1+\frac{R}{\gamma \delta^{n+1}}\right)^{n} .
\end{aligned}
$$

Therefore,

$$
\widetilde{\beta}_{n} \leqslant C(n+1) \gamma^{n} \delta^{(n+1)^{2}}\left(1+\frac{R}{\gamma \delta^{n+1}}\right)^{n}
$$

Thus $\varlimsup_{n \rightarrow \infty} \widetilde{\beta}_{n}^{1 / n^{2}} \leqslant \delta$. Since $\delta>1$ was arbitrary, the proof of Step 2 is complete.

Step 3. The conclusion of the lemma holds.

Proof of Step 3. Let $\delta>1$ be fixed. Since $\psi(0)=0$ and $\psi(a) \geqslant a$, we see that $\gamma=\left\|\psi^{\prime}\right\|_{\infty} \geqslant$ 1. Thus using that $c_{j} \geqslant 1$, we may take a positive integer $l$ such that

$$
\delta^{n / 2}\left(1+\frac{R}{\gamma(\gamma \delta)^{m} \delta^{(n+1) / 4} c_{j}}\right)^{n} \leqslant \delta^{n} \quad \text { for each } m \geqslant l \text { and } n, j \geqslant 0 .
$$


Indeed, it is enough to take $l$ with $\delta^{l} \geqslant R /(\sqrt{\delta}-1)$. As in the proof of Step 2, there is $C>0$ such that, for $0 \leqslant k \leqslant l$, we have

$$
M_{n}\left(T_{\psi}^{k} f_{j}\right) \leqslant C c_{j}^{n+k+1} \delta^{k / 4}(n)_{k}(\gamma \delta)^{k(n+(k-1) / 2)} \delta^{n^{2} / 4} \quad \text { for each } n, j \geqslant 0 .
$$

We will prove that (3.24) also holds for each $k \geqslant l+1$. Suppose that (3.24) is true for an integer $k=m \geqslant l$. For $k=m$, we can rewrite $(3.24)$ as $M_{n}\left(T_{\psi}^{m} f_{j}\right) \leqslant c(n)_{m} \widehat{\beta}_{n}$, where $c=C c_{j}^{m+1} \delta^{m / 4}(\gamma \delta)^{m(m-1) / 2}$ and $\widehat{\beta}_{n}=c_{j}^{n}(\gamma \delta)^{m n} \delta^{n^{2} / 4}$. Applying Step 1, we have

$$
M_{n}\left(T_{\psi}^{m+1} f_{j}\right) \leqslant c(n)_{m+1} \gamma^{n} \widehat{\beta}_{n+1}\left(1+\frac{R}{\gamma \widehat{\beta}_{n+1}^{1 /(n+1)}}\right)^{n}
$$

which is equal to

$$
C c_{j}^{m+n+1} \delta^{(m+1) / 4}(n)_{m+1}(\gamma \delta)^{m(m-1) / 2} \gamma^{n}(\gamma \delta)^{m n+m} \delta^{n^{2} / 4} \delta^{n / 2}\left(1+\frac{R}{\gamma c_{j}(\gamma \delta)^{m} \delta^{(n+1) / 4}}\right)^{n} .
$$

Since $m \geqslant l$, we may use (3.23) to obtain

$$
\begin{aligned}
M_{n}\left(T_{\psi}^{m+1} f\right) & \leqslant C c_{j}^{m+n+1} \delta^{(m+1) / 4}(n)_{m+1}(\gamma \delta)^{m(m-1) / 2} \gamma^{n}(\gamma \delta)^{m n+m} \delta^{n^{2} / 4} \delta^{n} \\
& =C c_{j}^{n+m+1} \delta^{(m+1) / 4}(n)_{m+1}(\gamma \delta)^{m n+m(m-1) / 2+n+m} \delta^{n^{2} / 4} \\
& =C \delta^{(m+1) / 4}(n)_{m+1}(\gamma \delta)^{(m+1)(n+(m / 2))} \delta^{n^{2} / 4}
\end{aligned}
$$

which is (3.24) for $k=m+1$. Thus (3.24) holds for all non-negative integers $k, n$ and $j$. For $n=0$ and $j=k$, we find that (3.24) implies that $\left\|T_{\psi}^{k} f_{k}\right\|_{\infty} \leqslant C c_{k}^{k+1} \delta^{k / 4} k !(\gamma \delta)^{k(k-1) / 2}$. Since $c_{k}^{1 / k}$ tends to 1 , we obtain $\varlimsup_{k \rightarrow \infty}\left\|T_{\psi}^{k} f_{k}\right\|_{\infty}^{1 / k^{2}} \leqslant \sqrt{\gamma \delta}$. Since $\delta>1$ was arbitrary, it follows that $\varlimsup_{\lim _{k \rightarrow \infty}}\left\|T_{\psi}^{k} f_{k}\right\|_{\infty}^{1 / k^{2}} \leqslant \sqrt{\gamma}$, which is the required result. The proof of Step 3 and that of the statement of the lemma are complete.

Observe that the formula for the adjoint of $V_{\varphi}$ is

$$
\left(V_{\varphi}^{\star} f\right)(x)=\int_{\varphi_{-1}(x)}^{1} f(t) d t .
$$

which, as an operator, makes sense on $L^{p}[0,1]$ for $1 \leqslant p \leqslant \infty$. Indeed, the adjoint of $V_{\varphi}^{\star}$ acting on $L^{1}[0,1]$ is $V_{\varphi}$ acting on $L^{\infty}[0,1]$. The next lemma, which will be very useful, describes the behaviour of the supports of the iterates $\left\{V_{\varphi}^{n} f\right\}$ and $\left\{V_{\varphi}^{\star n} f\right\}$. The proof, which is straightforward, is omitted.

Lemma 3.11. Let $\varphi$ be a continuous strictly increasing self-map of $[0,1]$ with $\varphi(x)<x$ and $\varphi(1)=1$. Assume also that $f$ is in $L^{1}[0,1]$. Then we have

(a) $\inf \operatorname{supp}\left(V_{\varphi} f\right)=\varphi_{-1}(\inf \operatorname{supp}(f))$;

(b) $\sup \operatorname{supp}\left(V_{\varphi} f\right) \in\left\{1, \varphi_{-1}(\sup \operatorname{supp}(f))\right\}$;

(c) $\inf \operatorname{supp}\left(V_{\varphi}^{\star} f\right) \in\{0, \varphi(\inf \operatorname{supp}(g))\}$;

(d) $\sup \operatorname{supp}\left(V_{\varphi}^{\star} g\right)=\varphi(\sup \operatorname{supp}(g))$;

(e) $\sup \operatorname{supp}\left(V_{\varphi}^{n} f\right)$ tends to 1 and $\inf \operatorname{supp}\left(V_{\varphi}^{\star n} g\right)$ tends to 0 as $n$ tends to $\infty$.

When dealing with supercyclicity of $V_{\varphi}$ in Section 5 , we shall need special dense subsets of $\mathcal{C}_{0}[0,1]$. For each $0<a<1$, we set $\mathcal{C}_{a}=\left\{f \in \mathcal{C}_{0}[0,1]\right.$ such that $\left.\sup \operatorname{supp}(f) \leqslant a\right\}$. We have the following lemma. 
LEMMA 3.12. Let $\varphi$ be a continuous strictly increasing self-map of $[0,1]$ with $\varphi(x)<x$ for $0<x<1$ and $\varphi(1)=1$. Assume that $0<a<1$. Then $Z=\operatorname{span}\left(\bigcup_{n=0}^{\infty} V_{\varphi}^{n}\left(\mathcal{C}_{a}\right)\right)$ is dense in $\mathcal{C}_{0}[0,1]$

Proof. It is enough to prove that $Z$ is dense in $L^{2}[0,1]$. Indeed, once this is proved, the result follows because $V_{\varphi}$ acting from $L^{2}[0,1]$ into $\mathcal{C}_{0}[0,1]$ is bounded with dense range and the image of a dense set under an operator with dense range is itself dense and $Z$ is invariant under $V_{\varphi}$.

Thus assume that $Z$ is not dense in $L^{2}[0,1]$. Then there is a non-zero $g$ in $L^{2}[0,1]$ such that $\left\langle V_{\varphi}^{n} f, g\right\rangle=\left\langle f, V_{\varphi}^{\star n} g\right\rangle=0$ for each $f$ in $\mathcal{C}_{a}$ and for each $n \geqslant 0$. This means that inf $\operatorname{supp}\left(V_{\varphi}^{\star n} g\right) \geqslant$ $a$ for each $n \geqslant 0$. Now, by Lemma 3.11, we have inf $\operatorname{supp}\left(V_{\varphi}^{\star n} g\right)$ tends to 0 as $n$ tends to $\infty$, which is a contradiction.

For $0<a<1$, we shall write

$$
\mathcal{F}_{a}=\left\{f \in \mathcal{E}_{a} \text { such that } \varlimsup_{n \rightarrow \infty}\left(M_{n}(f)\right)^{1 / n^{2}} \leqslant 1\right\},
$$

where $\mathcal{E}_{a}$ is as defined in (3.17); that is, $f$ belongs to $\mathcal{F}_{a}$ if and only if $f$ belongs to $\mathcal{C}^{\infty}[0,1]$, $\operatorname{supp}(f) \subseteq[0, a]$ and the restriction of $f$ to $[0, a]$ belongs to $\mathcal{F}[0, a]$.

Lemma 3.13. Let $\psi$ be analytic from $[0, a]$ into $[0,1]$, where $0<a<1$, with $\psi(0)=0$ and $\psi(a) \geqslant a$. Let $T_{\psi}$ be the operator on $\mathcal{E}_{a}$ defined in (3.18) and $C_{\psi}$ be the operator on $\mathcal{E}_{a}$ defined as

$$
\left(C_{\psi} f\right)(x)= \begin{cases}f(\psi(x)) & \text { if } x \in[0, a] \\ 0 & \text { if } x \in(a, 1]\end{cases}
$$

Then $\mathcal{F}_{a}$ is invariant both under $C_{\psi}$ and $T_{\psi}$.

Proof. Let $\gamma>1$ be fixed. If $f$ is in $\mathcal{F}_{a}$, then there is $c \geqslant 1$ such that $M_{n}(f) \leqslant c^{n} \gamma^{n^{2}}$ for each positive integer $n$. Since $\psi$ is analytic, by (3.13), the value $R=\sup _{n \geqslant 2}\left(M_{n}(\psi) / \gamma\right)^{1 /(n-1)}$ is finite. Now, from Lemma 3.9 , it follows that

$$
M_{n}\left(C_{\psi} f\right) \leqslant \sum_{n=k_{1}+\ldots+n k_{n}} \frac{\left(k_{1}+\ldots+k_{n}\right) !}{k_{1} ! \ldots k_{n} !} M_{k_{1}+\ldots+k_{n}}(f)\left(M_{1}(\psi)\right)^{k_{1}} \ldots\left(M_{n}(\psi)\right)^{k_{n}} .
$$

Using that $M_{m}(\psi) \leqslant \gamma R^{m-1}$ and $M_{k}(f) \leqslant c^{k} \gamma^{k^{2}}$, we obtain

$$
\begin{aligned}
M_{n}\left(C_{\psi} f\right) & \leqslant \sum_{n=k_{1}+\ldots+n k_{n}} \frac{\left(k_{1}+\ldots+k_{n}\right) !}{k_{1} ! \ldots k_{n} !} c^{k_{1}+\ldots+k_{n}} \gamma^{\left(k_{1}+\ldots+k_{n}\right)^{2}} \gamma^{k_{1}}(\gamma R)^{k_{2}} \ldots\left(\gamma R^{n-1}\right)^{k_{n}} \\
& \leqslant R^{n} \sum_{n=k_{1}+\ldots+n k_{n}} \frac{\left(k_{1}+\ldots+k_{n}\right) !}{k_{1} ! \ldots k_{n} !}\left(\frac{c \gamma^{n+1}}{R}\right)^{k_{1}+\ldots+k_{n}} .
\end{aligned}
$$

Upon applying (3.20), we obtain $M_{n}\left(C_{\psi} f\right) \leqslant R^{n-1} c \gamma^{n+1}\left(1+c \gamma^{n+1} / R\right)^{n-1} \leqslant\left(R+c \gamma^{n+1}\right)^{n}$. Therefore, it follows that $\varlimsup_{n \rightarrow \infty}\left(M_{n}\left(C_{\psi} f\right)\right)^{1 / n^{2}} \leqslant \gamma$. Since $\gamma>1$ was arbitrary, we see that $\varlimsup_{n \rightarrow \infty}\left(M_{n}\left(C_{\psi} f\right)\right)^{1 / n^{2}} \leqslant 1$ and, therefore, $C_{\psi} f$ belongs to $\mathcal{F}_{a}$. Finally, it is clear that $\mathcal{F}_{a}$ is also invariant under the differentiation operator $D f=f^{\prime}$. Since $T_{\psi}=C_{\psi} D$, the result follows.

The next lemma is needed not only to prove Theorem 3.6, but also to show the non-cyclicity of certain Volterra composition operators in Section 5. 
Lemma 3.14. Let $\varphi$ be a continuous strictly increasing self-map of $[0,1]$ with $\varphi(x)<x$ for $0<x<1$ and $\varphi(1)=1$. Assume also that $\varphi$ is analytic on $\left[0, \varphi_{-1}(a)\right]$, where $0<a<1$, with $\varphi^{\prime}(x)>0$ for $0 \leqslant x \leqslant \varphi_{-1}(a)$. Then $\mathcal{F}_{a}$ is contained in $V_{\varphi}^{\infty}\left(\mathcal{C}_{0}[0,1]\right)$ and $\overline{\lim }_{n \rightarrow \infty}\left\|V_{\varphi}^{-n} f\right\|_{\infty}^{1 / n^{2}} \leqslant$ $\sqrt{\gamma}$ for each $f \in \mathcal{F}_{a}$, where $\gamma=\max _{\left[0, \varphi^{-1}(a)\right]} 1 / \varphi^{\prime}$.

Proof. We set $\psi=\varphi_{-1}$ for the inverse of $\varphi$. Clearly, $\psi$ is analytic on $[0, a]$ and $\max _{[0, a]}\left|\psi^{\prime}\right|=\gamma$. It is easy to check that

$$
(S f)(x)= \begin{cases}\frac{f^{\prime}(\psi(x))}{\varphi^{\prime}(\psi(x))} & \text { if } x \in(0, a), \\ 0 & \text { otherwise }\end{cases}
$$

acts from $\mathcal{E}_{a}$ into $\mathcal{E}_{a}$ and that $V_{\varphi} S f=f$ for each $f$ in $\mathcal{E}_{a}$. Therefore, it follows that $\mathcal{F}_{a} \subset \mathcal{E}_{a} \subset$ $V_{\varphi}^{\infty}\left(\mathcal{C}_{0}[0,1]\right)$ and the operator $S$ defined in $(3.26)$ coincides with the restriction to $\mathcal{E}_{a}$ of $V_{\varphi}^{-1}$ acting on $V_{\varphi}^{\infty}\left(\mathcal{C}_{0}[0,1]\right)$.

Now consider the operator $T_{\psi}$ acting on $\mathcal{E}_{a}$ as defined in (3.18). One easily sees that $C_{\psi} S f=$ $T_{\psi} C_{\psi} f$ for each $f$ in $\mathcal{E}_{a}$, where $C_{\psi}$ is defined as $\left(C_{\psi} f\right)(x)=f(\psi(x))$. Hence $C_{\psi} S^{n} f=T_{\psi}^{n} C_{\psi} f$ for each $f$ in $\mathcal{E}_{a}$ and $n \geqslant 0$. Thus

$$
\left\|V_{\varphi}^{-n} f\right\|_{\infty}=\left\|S^{n} f\right\|_{\infty}=\left\|C_{\psi} S^{n} f\right\|_{\infty}=\left\|T_{\psi}^{n} C_{\psi} f\right\|_{\infty} \quad \text { for } f \in \mathcal{E}_{a} \text { and } n \geqslant 0 .
$$

Now, if $f$ belongs to $\mathcal{F}_{a}$, then, by Lemma 3.13, we have that $C_{\psi} f$ belongs to $\mathcal{F}_{a}$. Hence $\varlimsup_{n \rightarrow \infty}\left(M_{n}\left(C_{\psi} f\right)\right)^{1 / n^{2}} \leqslant 1$. Applying Lemma 3.10 with $c_{n}=1$ and $f_{n}=C_{\psi} f$ for each $n \geqslant 0$, we obtain $\varlimsup_{n \rightarrow \infty}\left\|T_{\psi}^{n} C_{\psi} f\right\|_{\infty}^{1 / n^{2}} \leqslant \sqrt{\gamma}$. Therefore, using (3.27), we have $\varlimsup_{n \rightarrow \infty}\left\|V_{\varphi}^{-n} f\right\|_{\infty}^{1 / n^{2}} \leqslant$ $\sqrt{\gamma}$, which is the required conclusion.

Now, we have all necessary tools to prove Theorem 3.6.

Proof of Theorem 3.6. One easily checks that $F_{b}$ is linear and that $V_{\varphi}\left(F_{b}\right)$ and $V_{\varphi}^{-1}\left(F_{b}\right)$ are contained in $F_{b}$, which implies that $V_{\varphi}\left(F_{b}\right)=F_{b}=V_{\varphi}^{-1}\left(F_{b}\right)$. Thus we need only prove that $F_{b}$ is dense in $\mathcal{C}_{0}[0,1]$.

Set $\psi=\varphi_{-1}$ for the inverse of $\varphi$. Since $b>1 / \varphi^{\prime}(0)$, we may choose $0<a<1$ such that $\varphi$ is analytic on $\left[0, \varphi_{-1}(a)\right]$ and $\varphi^{\prime}(x)>0$ for each $0 \leqslant x \leqslant \varphi_{-1}(a)$ and $\gamma=\max _{\left[0, \varphi_{-1}(a)\right]} 1 /\left|\varphi^{\prime}\right|=$ $\max _{[0, a]}\left|\psi^{\prime}\right| \leqslant b$. By Lemma 3.14, we have $\mathcal{F}_{a} \subset V_{\varphi}^{\infty}\left(\mathcal{C}_{0}[0,1]\right)$ and $\overline{\lim }_{n \rightarrow \infty}\left\|V_{\varphi}^{n} f\right\|_{\infty}^{1 / n^{2}} \leqslant \sqrt{\gamma} \leqslant$ $\sqrt{b}$ for each $f \in \mathcal{F}_{a}$. Thus we find that $\mathcal{F}_{a} \subset F_{b}$. Since $F_{b}$ is invariant under $V_{\varphi}$, we find that span $\left(\bigcup_{n=0}^{\infty} V_{\varphi}^{n}\left(\mathcal{F}_{a}\right)\right) \subseteq F_{b}$. By Lemma 3.8, we know that $\mathcal{F}_{a}$ is dense in the subspace $\mathcal{C}_{a}$ of functions in $\mathcal{C}_{0}[0,1]$ that vanish on $[a, 1]$. Hence, since $V_{\varphi}$ is bounded, it follows that $\overline{\operatorname{span}}\left(\bigcup_{n=0}^{\infty} V_{\varphi}^{n}\left(\mathcal{C}_{a}\right)\right) \subseteq \bar{F}_{b}$, where the closures are taken in $\mathcal{C}_{0}[0,1]$. We may conclude from Lemma 3.12 that the left-hand side in the latter inclusion coincides with $\mathcal{C}_{0}[0,1]$ and, therefore, $F_{b}$ is dense in $\mathcal{C}_{0}[0,1]$. The proof is complete.

\subsection{Orbits of $V_{\varphi}$ : lower estimate}

We begin with the following lemma that provides a lower estimate for orbits of $V_{\varphi}$.

THEOREM 3.15. Let $\varphi$ be a continuous strictly increasing self-map of $[0,1]$ with $\varphi(x)<x$ for $0<x<1$ and analytic at 1 with $\varphi(1)=1$. Then, for each non-zero $f$ in $L^{1}[0,1]$, we have

$$
\varliminf_{n \rightarrow \infty}\left\|V_{\varphi}^{n} f\right\|_{1}^{1 / n^{2}} \geqslant \frac{1}{\sqrt{\varphi^{\prime}(1)}} .
$$


Proof. Recall, from $[\mathbf{1 5}, \S 2]$, that the adjoint $V_{\varphi}^{\star}$ that acts on $L^{\infty}[0,1]$ is $V_{\varphi}^{\star}=U V_{\phi} U$, where $U$ is the involutive isometry defined by $(U g)(x)=g(1-x)$ and $\phi(x)=1-\varphi_{-1}(1-x)$. Since $\varphi$ is analytic at 1 , so is $\psi=\phi^{-1}$ at 0 . Take $\gamma>\psi^{\prime}(0) \geqslant 1$ and let $0<a<1$ be such that $\psi$ is analytic on $[0, a]$ and $\sup _{[0, a]} \psi^{\prime} \leqslant \gamma$.

By Lemma 3.11, we have that $\sup \operatorname{supp}\left(V_{\varphi}^{k} g\right)$ tends to 1 as $k$ tends to $\infty$ for each non-zero $g$ in $L^{1}[0,1]$. Therefore, for each non-zero $g$ in $L^{1}[0,1]$, we have $\sup \operatorname{supp}\left(V_{\varphi}^{k} g\right)>1-a$ for all $k$ large enough. Observe also that, for any $k \geqslant 1$ and for any non-zero $g$ in $L^{1}[0,1]$, the inequality in (3.28) is satisfied for $f=g$ if and only if it is satisfied for $f=V_{\varphi}^{k} g$. Since the range of $V_{\varphi}$ is contained in $\mathcal{C}_{0}[0,1]$, it is enough to show the inequality in $(3.28)$ for each $f$ in $\mathcal{C}_{0}[0,1]$ with $\sup \operatorname{supp}(f)>1-a$.

Thus assume that $f$ in $\mathcal{C}_{0}[0,1]$ has $\sup \operatorname{supp}(f)>1-a$. We may take $1-a<b<1$ and $\delta>0$ such that $1-a<b-\delta<b+\delta \leqslant 1$ and $f(b) \neq 0$. By Lemma $3.8, \mathcal{F}^{+}[1-b-\delta, 1-b+\delta]$ is dense in $\mathcal{C}_{00}^{+}[1-b-\delta, 1-b+\delta]$. In particular, there is $g_{1}$ in $\mathcal{F}[1-b-\delta, 1-b+\delta]$ such that $g_{1}(x) \geqslant 0$ for each $1-b-\delta \leqslant x \leqslant 1-b+\delta$ and

$$
\int_{1-b-\delta}^{1-b+\delta} g_{1}(x) d x=1
$$

We may think of $g_{1}$ as defined on the whole real line, by just making $g_{1}$ equal to 0 outside of $[1-b-\delta, 1-b+\delta]$. Now, consider $g_{n}(x)=n g_{1}(n x-(1-b)(n-1))$ for $n \geqslant 1$. In this way, $\left\{g_{n}\right\}_{n \geqslant 1}$ is a positive summability kernel at 0 (see [12, pp. 9-10]). Since $\operatorname{supp}\left(g_{n}\right) \subseteq[1-b-$ $\delta / n, 1-b+\delta / n] \subset[0, a]$, we may regard $\left\{g_{n}\right\}$ as a sequence in $\mathcal{E}_{a}$. Now, set $f_{n}(x)=g_{n}(\psi(x))$ and consider $R=\sup _{n \geqslant 2}\left(M_{n}(\psi) / \gamma\right)^{1 /(n-1)}$, where $M_{n}(\psi)=\sup _{[0, a]}\left|\psi^{(n)}\right| / n$ !. By Lemma 3.9 we find that

$$
M_{n}\left(f_{k}\right) \leqslant k \sum_{n=k_{1}+\ldots+n k_{n}} \frac{\left(k_{1}+\ldots+k_{n}\right) !}{k_{1} ! \ldots k_{n} !} M_{k_{1}+\ldots+k_{n}}\left(g_{1}\right)\left(M_{1}(\psi)\right)^{k_{1}} \ldots\left(M_{n}(\psi)\right)^{k_{n}} k^{k_{1}+\ldots+k_{n}} .
$$

Since $M_{n}(\psi) \leqslant \gamma R^{n-1}$, setting $\alpha_{n}=\max _{0 \leqslant j \leqslant n} M_{j}\left(g_{1}\right)$, we have that

$$
\begin{aligned}
M_{n}\left(f_{k}\right) & \leqslant k R^{n} \sum_{n=k_{1}+\ldots+n k_{n}} \frac{\left(k_{1}+\ldots+k_{n}\right) !}{k_{1} ! \ldots k_{n} !} M_{k_{1}+\ldots+k_{n}}\left(g_{1}\right)\left(\frac{k \gamma}{R}\right)^{k_{1}+\ldots+k_{n}} \\
& \leqslant k R^{n} \alpha_{n} \sum_{n=k_{1}+\ldots+n k_{n}} \frac{\left(k_{1}+\ldots+k_{n}\right) !}{k_{1} ! \ldots k_{n} !}\left(\frac{k \gamma}{R}\right)^{k_{1}+\ldots+k_{n}}
\end{aligned}
$$

Applying (3.20), we obtain $M_{n}\left(f_{k}\right) \leqslant \alpha_{n} k^{2} R^{n-1}(1+k \gamma / R)^{n-1}=\alpha_{n} k^{2}(R+k \gamma)^{n-1} \leqslant \alpha_{n}(R+$ $k \gamma)^{n+1}$. Since $g_{1}$ belongs to $F_{\psi(a)}$, we have $\overline{\lim }_{n \rightarrow \infty} \alpha_{n}^{1 / n^{2}} \leqslant 1$ and, therefore, all the hypotheses of Lemma 3.10 with $c_{k}=R+k \gamma$ are fulfilled. Thus

$$
\varlimsup_{n \rightarrow \infty}\left\|T_{\psi}^{n} f_{n}\right\|_{\infty}^{1 / n^{2}} \leqslant \sqrt{\gamma}
$$

where $T_{\psi}$ is as in (3.18). Since $V_{\phi}^{-1} f=C_{\phi} T_{\psi} C_{\phi}^{-1} f=C_{\phi} T_{\psi} C_{\psi} f$ for each $f \in \mathcal{E}_{a}$, we find that $g_{n}=V_{\phi}^{n} C_{\phi} T_{\psi}^{n} C_{\psi} g_{n}=V_{\phi}^{n} C_{\phi} T_{\psi}^{n} f_{n}$. Using that $U$ is involutive and $V_{\varphi}^{\star}=U V_{\phi} U$, we see that $U g_{n}=V_{\varphi}^{\star n} U C_{\phi} T_{\psi}^{n} f_{n}$. Therefore,

$$
\left\|V_{\varphi}^{n} f\right\|_{1} \geqslant \frac{\left|\left\langle V_{\varphi}^{n} f, U C_{\phi} T_{\psi}^{n} f_{n}\right\rangle\right|}{\left\|U C_{\phi} T_{\psi}^{n} f_{n}\right\|_{\infty}} \geqslant \frac{\left|\left\langle f, V_{\varphi}^{\star n} U C_{\phi} T_{\psi}^{n} f_{n}\right\rangle\right|}{\left\|U C_{\phi} T_{\psi}^{n} f_{n}\right\|_{\infty}}=\frac{\left|\left\langle f, U g_{n}\right\rangle\right|}{\left\|T_{\psi}^{n} f_{n}\right\|_{\infty}} .
$$


Since $\left\{g_{n}\right\}$ is a positive summability kernel at $1-b$, then so is $\left\{U g_{n}\right\}$ at $b$. Therefore, $\left|\left\langle f, U g_{n}\right\rangle\right|$ converges to $|f(b)| \neq 0$. Thus,

$$
\left\|V_{\varphi}^{n} f\right\|_{1} \geqslant \frac{|f(b)|}{\left\|T_{\psi}^{n} f_{n}\right\|_{\infty}}(1+o(1)) \quad \text { as } n \rightarrow \infty
$$

which along with (3.29) implies that $\underline{\lim }_{n \rightarrow \infty}\left\|V_{\varphi}^{n} f\right\|_{p}^{1 / n^{2}} \geqslant 1 / \sqrt{\gamma}$. Since $\gamma>\psi^{\prime}(0)=\varphi^{\prime}(1)$ was arbitrary, the result follows.

From Lemma 3.15 and Corollary 3.4, we immediately have the following theorem.

THEOREM 3.16. Let $\varphi$ be a continuous strictly increasing self-map of $[0,1]$ with $\varphi(1)=1$ and $\varphi(x)<x$ for $0<x<1$. Assume also that $\varphi$ is analytic at 1 and differentiable at 0 with $\varphi^{\prime}(0)=0$. Then, for each non-zero $f$ in $L^{p}[0,1], 1 \leqslant p \leqslant \infty$, we have $\lim _{n \rightarrow \infty}\left\|V_{\varphi} f\right\|_{p}^{1 / n^{2}}=$ $1 / \sqrt{\varphi^{\prime}(1)}$

\section{Dense generalized kernels}

In the next section, we will prove that if $\varphi$ is continuous, strictly increasing and satisfies $\varphi(x)<x$ for $0<x \leqslant 1$, then $V_{\varphi}$ is supercyclic and $I+V_{\varphi}$ is hypercyclic when $V_{\varphi}$ acts on $L^{p}[0,1], 1 \leqslant p<\infty$, or on $\mathcal{C}_{0}[0,1]$. To do this, we adopt a general point of view. We will show that if $T$ is a continuous operator on a separable complete metrizable topological vector space $X$ such that the span of the union of $\operatorname{ker} T^{n} \cap T^{n}(X)$ is dense in $X$, then the operator $I+T$ is hypercyclic. We will also show that in such a case $T$ is supercyclic. This general point of view causes minimal extra effort and avoids the repetition of some arguments.

Recall that an $\mathcal{F}$-space is a complete metrizable topological vector space. The space of continuous linear operators on a topological vector space $X$ is denoted by $\mathcal{L}(X)$.

Recall that a continuous operator $T$ acting on a topological vector space $X$ is said to be hypercyclic if there is $x$ in $X$ such that the orbit of $x$ under $T$, that is, $\left\{T^{n} x\right\}_{n \geqslant 0}$, is dense in $X$ and it is said to be supercyclic if there is $x$ in $X$ such that the projective orbit $\left\{\lambda T^{n}\right.$ such that $\left.\lambda \in \mathbb{C}, n=0,1, \ldots\right\}$ is dense in $X$. We say that $T$ is strongly hereditarily hypercyclic if, for every subsequence $\left\{n_{k}\right\}$ of positive integers, there is $x$ such that $\left\{T^{n_{k}} x\right\}$ is dense in $X$. Later concept has been used in [7]. Similarly, we can define strongly hereditarily supercyclic operators.

A bounded operator $T$ acting on a locally convex topological vector space is called weakly hypercyclic or weakly supercyclic if it is hypercyclic or supercyclic with respect to the weak topology. Mazur's theorem asserts that the norm closure and the weak closure of convex sets coincide, and hence weakly supercyclic operators are cyclic. Hypercyclic and supercyclic operators have been intensely studied during the last few decades (see the surveys $[\mathbf{9}, \mathbf{1 6}]$ and references therein).

Let $\ell^{p}, 1 \leqslant p<\infty$, denote the Banach space of complex sequences that have $p$-summable modulus. Let $\left\{e_{n}\right\}_{n \geqslant 0}$ be the canonical basis of $\ell^{p}$, where $1 \leqslant p<\infty$. Given a bounded sequence $\left\{w_{n}\right\}$ of non-zero complex numbers, the backward weighted shift with weight sequence $\left\{w_{n}\right\}$ is defined by $T e_{0}=0$ and $T e_{n}=w_{n} e_{n-1}$ for $n \geqslant 1$.

The next theorem, due to Salas [19], is one of the most important results on hypercyclicity for a fixed operator.

Theorem 4.1 (Salas' theorem). Let $T$ be a backward weighted shift on $\ell^{2}$. Then the operator $I+T$ is hypercyclic. 
The next theorem extends Salas' theorem in several directions.

Theorem 4.2. Let $T$ be a continuous operator on a separable $\mathcal{F}$-space $X$ such that

$$
\operatorname{ker}^{\dagger} T=\operatorname{span}\left(\bigcup_{n=1}^{\infty}\left(T^{n}(X) \cap \operatorname{ker} T^{n}\right)\right)
$$

is dense in $X$. Then $I+T$ is (strongly hereditarily) hypercyclic.

Recall that the generalized kernel of an operator $T$ is the space $\operatorname{ker}^{\star} T=\bigcup_{n=1}^{\infty} \operatorname{ker} T^{n}$. It is worth mentioning that the space $\operatorname{ker}^{\dagger} T$ is contained in $T(X)$ as well as in $\operatorname{ker}^{\star} T$. Thus, any operator with dense $\operatorname{ker}^{\dagger} T$ has dense range and dense generalized kernel. Obviously, if $T$ is a (unilateral) backward weighted shift on $\ell^{p}$, then $\operatorname{ker}^{\star} T=\operatorname{ker}^{\dagger} T$ is the space of sequences with finite support, which is dense in $\ell^{p}$, where $1 \leqslant p<\infty$. Hence Theorem 4.2 implies Salas' theorem. It is also worth noting that if $T\left(\operatorname{ker} T^{n+1}\right)$ is dense in $\operatorname{ker} T^{n}$ for each positive integer $n$, then $\operatorname{ker}^{\dagger} T$ is dense in $\operatorname{ker}^{\star} T$. Thus, we have the following corollary.

Corollary 4.3. Let $T$ be a continuous operator on a separable $\mathcal{F}$-space $X$ such that $\operatorname{ker}^{\star} T$ is dense in $X$ and $T\left(\operatorname{ker} T^{n+1}\right)$ is dense in $\operatorname{ker} T^{n}$ for each positive integer $n$. Then $I+T$ is (strongly hereditarily) hypercyclic.

The advantage of the above corollary is that it is much easier to check that $T\left(\operatorname{ker} T^{n+1}\right)$ is dense in $\operatorname{ker} T^{n}$.

A generalized backward shift is a continuous operator $T$ on a topological vector space $X$ such that $\operatorname{ker} T$ is one-dimensional and $\operatorname{ker}^{\star} T$ is dense in $X$. A dimension argument shows immediately that if $T$ is a generalized backward shift, then $\operatorname{ker} T^{n}$ is $n$-dimensional and $T\left(\operatorname{ker} T^{n+1}\right)=\operatorname{ker} T^{n}$ for each positive integer $n$. From Corollary 4.3, we clearly have the following corollary.

Corollary 4.4. Let $X$ be a separable $\mathcal{F}$-space and $T$ in $\mathcal{L}(X)$ be a generalized backward shift. Then $I+T$ is (strongly hereditarily) hypercyclic.

REMARK. The fact that $I+T$ is hypercyclic for a generalized backward shift $T$ on a separable $\mathcal{F}$-space also follows from Salas' theorem by means of a quasisimilarity argument, as already observed by several authors (see, for instance, $[\mathbf{8}]$ ).

To prove Theorem 4.2, we need some preparation.

\subsection{A density criterion}

Recall that a topological space $X$ is called a Baire space if, for each first category set $A \subset X$, its complement $X \backslash A$ is dense in $X$. According to the classical Baire theorem, complete metric spaces are Baire. We need the following easy proposition.

Proposition 4.5. Let $X$ and $Y$ be Baire topological spaces, where $Y$ is second countable. Let $\left\{T_{n}\right\}_{n \geqslant 0}$ be a sequence of continuous maps from $X$ to $Y$. Let $\Sigma$ be the set of $(x, y) \in X \times Y$ for which there exists a sequence $\left\{x_{n}\right\}_{n} \geqslant 0$ in $X$ such that $x_{n} \rightarrow x$ and $T_{n} x_{n} \rightarrow y$. If $\Sigma$ is dense in $X \times Y$, then, for any subsequence $\left\{n_{k}\right\}_{k} \geqslant 0$, there is $x$ such that $\left\{T_{n_{k}} x\right\}_{k \geqslant 0}$ is dense in $X$. 
Proof. Since $\Sigma$ is dense in $X \times Y$, it is enough to apply Theorem 1 in [9, p. 348].

\subsection{Invertible matrices}

To prove Theorem 4.2, we need to show that certain matrices are invertible. For each pair of positive integers $n$ and $k$, consider the $n$-square matrix

$$
M_{n, k}=\left(\frac{(k+n-l) !}{(k+n-l+j-1) !}\right)_{1 \leqslant j, l \leqslant n} .
$$

Lemma 4.6. For each pair $n$ and $k$ of positive integers, we have

$$
\operatorname{det} M_{n, k}=\frac{(n-1) ! k !(k+1) !}{(k+n-1) !(k+n) !} \operatorname{det} M_{n-1, k+2} .
$$

Proof. It is clear that (4.1) holds for $n=2$. Thus suppose that $n \geqslant 3$. Subtracting from each column of $M_{n, k}$, except the first, the previous one, we see that

$$
\operatorname{det} M_{n, k}=\operatorname{det}\left(\begin{array}{cccc}
\frac{(k+n-1) !}{(k+n) !} & 0 & \ldots & 0 \\
\vdots & & & \\
\frac{(k+n-1) !}{(k+2 n-2) !} & & &
\end{array}\right)
$$

where

$$
N_{n, k}=\left(j \frac{(k+n-l-1) !}{(k+n-l+j) !}\right)_{1 \leqslant j, l \leqslant n-1} .
$$

Thus $\operatorname{det} M_{n, k}=\operatorname{det} N_{n, k}$. Now, dividing each $j$ th row of $N_{n, k}$ by $j$ and multiplying each $l$ th column with $(k+n-l+1) ! /(k+n-l-1)$ !, we obtain $M_{n-1, k+2}$. Hence

$$
\operatorname{det} M_{n, k}=\operatorname{det} M_{n-1, k+2} \prod_{\substack{j=1 \\ l=1}}^{n-1} \frac{j(k+n-l-1) !}{(k+n-l+1) !}=\frac{(n-1) ! k !(k+1) !}{(k+n-1) !(k+n) !} \operatorname{det} M_{n-1, k+2} .
$$

The result is proved.

Consider now the $n$-square matrix $A_{n}=(1 /(j+k-1) !)_{1 \leqslant j, k \leqslant n}$. The key lemma in the proof of Salas' theorem is [19, Lemma 3.1], which asserts that $A_{n}$ is invertible for $n=2^{k}$ with $k$ a positive integer. The latter is also used in $[\mathbf{1 3}]$ to prove that the operators in Salas' theorem do satisfy Kitai's criterion. Actually, $A_{n}$ is invertible for each positive integer $n$. Indeed, $\operatorname{det} A_{n}$ can be computed explicitly.

Lemma 4.7. For each positive integer, the matrix $A_{n}$ is invertible. Furthermore, $\operatorname{det} A_{1}=1$, $\operatorname{det} A_{2}=-1 / 12$ and

$$
\operatorname{det} A_{n}=\frac{(-1)^{(n-1) n / 2}}{(2 n-1) !}\left(\prod_{j=1}^{2 n-4} j !\right)\left(\prod_{j=n}^{2 n-3} j !^{-2}\right) \quad \text { for } n \geqslant 3
$$

Proof. Let $B_{n}$ be the matrix obtained from $A_{n}$ by reversing the order of the columns of $A_{n}$. Clearly, $\operatorname{det} A_{n}=(-1)^{(n-1) n / 2} \operatorname{det} B_{n}$. Multiplying the $j$ th column of $B_{n}$ with $(n-j+1)$ ! 
for $1 \leqslant j \leqslant n$, we obtain $M_{n, 1}$. Hence, $\operatorname{det} A_{n}=(-1)^{(n-1) n / 2} \operatorname{det} M_{n, 1} \prod_{j=1}^{n}(j !)^{-1}$ for each $n \geqslant 1$. Now, the result follows by applying $n-1$ times (4.1) and then simplifying.

Finally, for each pair of positive integers $m$ and $n$ with $m \geqslant 2 n$, we consider the $n$-square matrix

$$
B_{m, n}=\left(\left(\begin{array}{c}
m \\
k+j-1
\end{array}\right)\right)_{1 \leqslant j, k \leqslant n}
$$

where $\left(\begin{array}{c}m \\ k\end{array}\right)$ denotes the binomial coefficient.

Lemma 4.8. For each pair of positive integers $m$ and $n$ with $m \geqslant 2 n$, we have that $B_{m, n}$ is invertible. Furthermore, $\operatorname{det} B_{m, n}=\operatorname{det} A_{n} \prod_{j=-n}^{n}(m+j)^{n-|j|}$.

Proof. By multiplying the $j$ th column of $B_{m, n}$ with $(m-j) ! / m$ ! for $1 \leqslant j \leqslant n$, we obtain $P_{m, n}$, whose entries are $p_{1, k}=1 / k !, 1 \leqslant k \leqslant n$, and

$$
p_{j, k}=\frac{(m-k) !}{(k+j-1) !(m-k-j+1) !} \quad \text { for } j \geqslant 2 .
$$

Consider $Q_{m, n}$ obtained from $P_{m, n}$ by replacing the $j$ th row $P_{[j]}$ by $\sum_{l=0}^{j-1}\left(\begin{array}{c}j-1 \\ l\end{array}\right) P_{[l+1]}$. Clearly, $\operatorname{det} P_{m, n}=\operatorname{det} Q_{m, n}$. In addition, one easily checks that the entries of $Q_{m, n}$ are $q_{1, k}=1 / k$ !, $1 \leqslant k \leqslant n$, and

$$
q_{j, k}=\frac{(m+j-1) !}{m !(k+j-1) !} \text { for } j \geqslant 2 .
$$

Multiplying the $j$ th row of $Q_{m, n}$ with $m ! /(m+j-1)$ ! for $2 \leqslant j \leqslant n$, we arrive at $A_{n}$. Upon putting everything together, we obtain

$$
\operatorname{det} B_{m, n}=\left(\prod_{j=1}^{n} \frac{m !}{(m-j) !}\right)\left(\prod_{j=2}^{n} \frac{(m+j-1) !}{m !}\right) \operatorname{det} A_{n} .
$$

Simplifying, the required formula for $\operatorname{det} B_{m, n}$ follows.

\subsection{Proof of Theorem 4.2}

For $x$ in $\mathbb{C}^{n}, n \geqslant 1$, we denote by $x_{j}$ its $j$ th coordinate.

Lemma 4.9. Let $S$ in $\mathcal{L}\left(\mathbb{C}^{2 n}\right), n \geqslant 1$, be defined on the canonical basis $\left\{e_{i}: 1 \leqslant i \leqslant 2 n\right\}$ by $S e_{i}=e_{i-1}, 2 \leqslant i \leqslant 2 n$ and $S e_{1}=0$. Then, for each $m \geqslant 2 n$ and each $u$ and $v$ in $\mathbb{C}^{n}$, there exists a unique $x=x(m)$ in $\mathbb{C}^{2 n}$ such that:

(a) $x_{j}(m)=u_{j}$, for $1 \leqslant j \leqslant n$;

(b) $\left((I+S)^{m} x(m)\right)_{j}=v_{j}$, for $1 \leqslant j \leqslant n$.

Furthermore,

$$
\begin{aligned}
\left|x_{n+j}(m)\right|=O\left(m^{-j}\right) & \text { as } m \rightarrow \infty \text { for } 1 \leqslant j \leqslant n, \\
\left|\left((I+S)^{m} x(m)\right)_{n+j}\right| & =O\left(m^{-j}\right) \quad \text { as } m \longrightarrow \infty \text { for } 1 \leqslant j \leqslant n .
\end{aligned}
$$

Proof. For $y$ in $\mathbb{C}^{2 n}$ and $z$ in $\mathbb{C}^{n}$, we define $\widetilde{y}=\left(y_{n+1}, \ldots, y_{2 n}\right) \in \mathbb{C}^{n}$ and $\widehat{z}=$ $\left(z_{1}, \ldots, z_{n}, 0, \ldots, 0\right)$ in $\mathbb{C}^{2 n}$. Let also $w(m)$ in $\mathbb{C}^{n}$ be defined by $w_{j}(m)=v_{n-j+1}-((I+$ $\left.S)^{m} \widehat{u}\right)_{n-j+1}$ for $1 \leqslant j \leqslant n$. One easily sees that there is a unique $x(m)$ satisfying (a) and (b) if and only if the equation

$$
B_{m, n} \widetilde{x}=w(m),
$$


where $B_{m, n}$ is the matrix defined in the previous subsection, has a unique solution. Thus the first statement of the lemma follows from Lemma 4.8.

It remains to show that (4.2) and (4.3) also hold. To this end, first observe that $w_{j}(m)=$ $v_{n-j+1}-\sum_{l=0}^{j-1}\left(\begin{array}{c}m \\ l\end{array}\right) u_{n-j+1+l}$ for $1 \leqslant j \leqslant n$. Thus

$$
w_{j}^{m}=O\left(m^{j-1}\right) \quad \text { as } m \longrightarrow \infty \text { for } 1 \leqslant j \leqslant n .
$$

Now consider the $n$-diagonal matrix $D_{m, n}$ with entries $m^{j-1}, 1 \leqslant j \leqslant n-1$, in the main diagonal. An easy computation shows that $B_{m, n}=m D_{m, n} C_{m, n} D_{m, n}$, where $C_{m, n}=\left\{\gamma_{j, k}\right\}_{1 \leqslant j, k \leqslant n}$ has entries $\gamma_{1,1}=1$ and $\gamma_{j, k}=(1 /(j+k-1) !) \prod_{l=1}^{j+k-2}(1-l / m)$ for $(j, k) \neq(1,1)$. Since $B_{m, n}$ as well as $D_{m, n}$ are invertible, so is $C_{m, n}$ and (4.4) implies that $\widetilde{x}^{m}=B_{m, n}^{-1} w(m)=$ $m^{-1} D_{m, n}^{-1} C_{m, n}^{-1} D_{m, n}^{-1} w(m)$.

From (4.5), the sequence $\left\{D_{m, n}^{-1} w(m)\right\}_{m \geqslant 2 n}$ is bounded in $\mathbb{C}^{n}$. On the other hand, the sequence of invertible matrices $\left\{C_{m, n}\right\}_{m \geqslant 2 n}$ converges to the matrix $A_{n}$ defined in Subsection 4.2 , which is invertible by Lemma 4.7. Hence, the sequence $\left\{C_{m, n}^{-1}\right\}$ converges to $A_{n}^{-1}$ as $m$ tends to $\infty$ and, therefore, the sequence $\left\{C_{m, n}^{-1} D_{m, n}^{-1} w^{m}\right\}_{m \geqslant 2 n}$ is bounded in $\mathbb{C}^{n}$. Hence,

$$
x_{n+j}(m)=\widetilde{x}_{j}(m)=m^{-1}\left(D_{m, n}^{-1} C_{m, n}^{-1} D_{m, n}^{-1} w^{m}\right)_{j}, \quad 1 \leqslant j \leqslant n,
$$

satisfy (4.2). Finally, since $\left((I+S)^{m} x(m)\right)_{n+j}=\sum_{l=0}^{n-j}\left(\begin{array}{c}m \\ l\end{array}\right) x_{n+j+l}(m)$ for $1 \leqslant j \leqslant n$, the estimates in (4.3) follow from (4.2) and the result is proved.

Lemma 4.9 allows us to prove the following lemma.

Lemma 4.10. Let $T$ be a continuous operator on a topological vector space $X$. Assume that $x$ belongs to $T^{m}(X) \cap \operatorname{ker} T^{m}$, where $m$ is a positive integer. Then there exist sequences $\left\{u_{k}\right\}_{k \geqslant 0}$ and $\left\{v_{k}\right\}_{k \geqslant 0}$ in $X$ such that

$$
u_{k} \longrightarrow 0,(I+T)^{k} u_{k} \longrightarrow x, v_{k} \longrightarrow x \quad \text { and } \quad(I+T)^{k} v_{k} \longrightarrow 0 \text { as } n \longrightarrow \infty .
$$

Proof. If $x=0$, then it is enough to take $u_{k}=v_{k}=0$. Thus, assume that $x \neq 0$. We will show that the proof reduces to the operator $S$ defined in Lemma 4.9. Let $n$ be the smallest positive integer for which $T^{n} x=0$. In particular, $n \leqslant m$, which implies that $x$ belongs to $T^{n}(X)$. Thus we may choose $w$ in $X$ such that $T^{n} w=x$. We set $h_{j}=T^{2 n-j} w$ for $1 \leqslant j \leqslant$ $2 n$ and $Y=\operatorname{span}\left\{h_{1}, \ldots, h_{2 n}\right\}$. In particular, we have $T h_{j}=h_{j-1}, 2 \leqslant j \leqslant 2 n$, and $T h_{1}=$ $T^{2 n} h_{2 n}=T^{n} x=0$. Thus clearly, $Y$ is invariant under $T$. Since $h_{1}=T^{2 n-1} h_{2 n}=T^{n-1} x \neq 0$, it follows that $\operatorname{dim} Y \geqslant 2 n$ and, therefore, $\left\{h_{1}, \ldots, h_{2 n}\right\}$ is a basis of $Y$.

Let $J$ be the operator from $\mathbb{C}^{2 n}$ onto $Y$ defined by $J e_{k}=h_{k}, 1 \leqslant k \leqslant 2 n$. Clearly, $T$ acting on $Y$ is similar under $J$ to $S$ acting on $\mathbb{C}^{2 n}$, where $S$ is the operator defined on Lemma 4.9 . Now, $J^{-1} x=e_{n}$. Thus taking, $u=(0, \ldots, 0,1)$ in $\mathbb{C}^{n}$ and $v=(0, \ldots, 0)$, we find that there is a sequence $\left\{g_{k}\right\}_{k \geqslant 0}$ in $\mathbb{C}^{2 n}$ such that $g_{k} \rightarrow e_{n}$ and $(I+S)^{k} g_{k} \rightarrow 0$ as $k \rightarrow \infty$. Applying Lemma 4.9 with $u=(0, \ldots, 0,0)$ and $v=(0, \ldots, 0,1)$, we find that there is a sequence $\left\{f_{k}\right\}_{k \geqslant 0}$ in $\mathbb{C}^{2 n}$ such that $f_{k} \rightarrow 0$ and $(I+S)^{k} f_{k} \rightarrow e_{m}$ as $k \rightarrow \infty$. The result follows because two topological vector spaces of the same finite dimension are homeomorphic under any algebraic isomorphism between them.

Lemma 4.11. Let $T$ be a continuous operator on a topological vector space $X$. Assume that $x$ and $y$ belong to $\operatorname{ker}^{\dagger} T$. Then there exists a sequence $\left\{x_{k}\right\}$ in $X$ such that $x_{k} \rightarrow x$ and $(I+T) x_{k} \rightarrow y$ as $k \rightarrow \infty$. 
Proof. Let $\Sigma$ be the set of $(x, y)$ in $X \times X$ for which there is a sequence $\left\{x_{n}\right\}$ in $X$ such that $x_{n}$ tends to $x$ and $(I+T)^{n} x_{n}$ tends to $y$. By Lemma 4.10, we have $\operatorname{ker} T^{n} \cap T^{n}(X) \times\{0\} \subset \Sigma$ and $\{0\} \times \operatorname{ker} T^{n} \cap T^{n}(X) \subset \Sigma$ for each $n \geqslant 1$. On the other hand, it is clear that $\Sigma$ is a subspace of $X \times X$. Therefore, one immediately obtains that $\operatorname{ker}^{\dagger} T \times \operatorname{ker}^{\dagger} T \subseteq \Sigma$, which is what had to be proved.

Now we are ready to prove Theorem 4.2.

Proof of Theorem 4.2. Let $\Sigma$ be the set of $(x, y) \in X \times X$ for which there is $\left\{x_{n}\right\}$ in $X$ such that $x_{n} \rightarrow x$ and $(I+T)^{n} x_{n} \rightarrow y$. By Lemma 4.11, it follows that $\Sigma$ contains $\operatorname{ker}^{\dagger} T \times \operatorname{ker}^{\dagger} T$. Since $\operatorname{ker}^{\dagger} T$ is dense in $X$, we obtain that $\Sigma$ is dense in $X \times X$. According to Theorem 4.5, for each subsequence $\left\{n_{k}\right\}$ there is $x$ in $X$ such that $\left\{(I+T)^{n_{k}} x\right\}$ is dense in $X$, that is, $I+T$ is strongly hereditarily hypercyclic. The proof of Theorem 4.2 is complete.

\subsection{Supercyclicity}

To prove the supercyclicity of $V_{\varphi}$, we extend another result by Salas [20].

Proposition 4.12. Let $X$ be a separable $\mathcal{F}$-space and $T$ be in $\mathcal{L}(X)$. Assume also that $T$ has dense range and dense generalized kernel. Then $T$ is (strongly hereditarily) supercyclic.

The advantage of Proposition 4.12 over Corollary 2.8 in [20] is that we avoid the existence of the local inverse.

The next criterion for an operator to be strongly hereditarily supercyclic is analogous to one of the forms of the Supercyclicity Criterion (see [16]).

Theorem 4.13. Let $T$ be a continuous operator on an $\mathcal{F}$-space $X$ and $\left\{\lambda_{k}\right\}_{k \geqslant 0}$ be a sequence of non-zero complex numbers. Assume also that there exist dense subsets $E$ and $F$ of $X$ and mappings $S_{k}: F \rightarrow X$ such that $T^{k} S_{k} y \rightarrow y$ and $\lambda_{k}^{-1} S_{k} y \rightarrow 0$ for each $y \in F$ and $\lambda_{k} T^{k} x \rightarrow 0$ for each $x \in E$ as $k \rightarrow \infty$. Then, for any strictly increasing sequence $\left\{n_{k}\right\}_{k \geqslant 0}$ of positive integers, there exists $x \in X$ for which $\left\{\lambda_{n_{k}} T^{n_{k}} x: k \geqslant 0\right\}$ is dense in $X$.

Proof of Proposition 4.12. Let $d$ be a metric that induces the topology of $X$. Let $F$ be a dense countable subset of $X$. Since $T$ has dense range, we find that $T^{k}(X)$ is dense in $X$ for each $k \geqslant 0$. Hence, we may choose $S_{k}: F \rightarrow X$ such that $d\left(y, T^{k} S_{k} y\right)<2^{-k}$ for each $y$ in $F$ and each $k \geqslant 0$. Clearly, $T^{k} S_{k} y \rightarrow y$ for each $y$ in $F$. Since $F$ is countable and $X$ is metrizable, there is a sequence $\left\{\lambda_{n}\right\}$ of positive numbers such that $\lambda_{n}^{-1} S_{n} y \rightarrow 0$ as $n \rightarrow \infty$ for each $y$ in $F$. Finally, $E=\operatorname{ker}^{\star} T$ is dense in $X$ and, for each $y$ in $E$, we have $T^{n} y=0$ for all $n$ large enough and, therefore, $\lambda_{n} T^{n} y \rightarrow 0$ as $n \rightarrow \infty$. Thus all the hypotheses of Theorem 4.13 are fulfilled and we conclude that $T$ is strongly hereditarily supercyclic.

\section{Supercyclicity of $V_{\varphi}$ and hypercyclicity $I+V_{\varphi}$}

In this section we study the supercyclicity of $V_{\varphi}$ as well as the hypercyclicity of $I+V_{\varphi}$ acting on the spaces $L^{p}[0,1], 1 \leqslant p<\infty$. Since $V_{\varphi}$ is a contraction on $L^{p}[0,1], 1 \leqslant p<\infty$, it cannot be weakly hypercyclic.

The following easy proposition states that if $V_{\varphi}$ is weakly supercyclic, then $\varphi(x) \leqslant x$ almost everywhere. 
Proposition 5.1. Let $\varphi$ is a measurable self-map of $[0,1]$ with $\varphi(x)>x$ on a set of positive Lebesgue measure. Then $V_{\varphi}$ acting on $L^{p}[0,1], 1 \leqslant p<\infty$, is not weakly supercyclic.

Proof. A supercyclic compact operator on a Banach space must be quasinilpotent (see [10]) and the same is true for weakly supercyclic operators (the same argument works). By $[\mathbf{1 5}$, Corollary 2.2], the operator $V_{\varphi}$ is not quasinilpotent and the result follows.

In what follows, we will be considering only continuous symbols. The following lemmas describe the closure of the range of $V_{\varphi}$. We denote by $\overline{\operatorname{ran}}_{p} V_{\varphi}$ the closure of the range of $V_{\varphi}$ acting on $L^{p}[0,1]$ and, when acting on $\mathcal{C}[0,1]$ or $\mathcal{C}_{0}[0,1]$, it will be denoted by $\overline{\operatorname{ran}} V_{\varphi} \operatorname{or}_{\overline{\operatorname{ran}}} V_{\varphi}$, respectively.

Lemma 5.2. Let $\varphi$ be a continuous self-map of $[0,1]$. Assume that $V_{\varphi}$ acts on $\mathcal{C}[0,1]$. If $\varphi$ is not strictly monotone, then the codimension of $\overline{\operatorname{ran}} V_{\varphi}$ is infinite. If $\varphi$ is strictly monotone and $\varphi(0) \neq 0, \varphi(1) \neq 0$, then $\overline{\operatorname{ran}} V_{\varphi}=\mathcal{C}[0,1]$. If $\varphi$ is strictly monotone and $\varphi(0)=0$, then $\overline{\operatorname{ran}} V_{\varphi}=\{f \in \mathcal{C}[0,1]: f(0)=0\}$. Finally, if $\varphi$ is strictly monotone and $\varphi(1)=0$, then $\overline{\operatorname{ran}} V_{\varphi}=$ $\{f \in \mathcal{C}[0,1]: f(1)=0\}$.

Proof. If $\varphi$ is not strictly monotone, then $A=\left\{(t, s) \in[0,1]^{2}: t<s\right.$ and $\left.\varphi(t)=\varphi(s)\right\}$ is infinite. Since $\overline{\operatorname{ran}} V_{\varphi} \subseteq\{f \in \mathcal{C}[0,1]: f(t)=f(s)$ for each $(t, s) \in A\}$ and the last space has infinite codimension, $\overline{\mathrm{ran}} V_{\varphi}$ has infinite codimension in $\mathcal{C}[0,1]$.

The description of $\overline{\operatorname{ran}} V_{\varphi}$ in the case when $\varphi$ is strictly monotone follows from the decomposition $V_{\varphi}=C_{\varphi} V$ and the fact that the closure of the range of the Volterra operator acting on $\mathcal{C}[0,1]$ is $\mathcal{C}_{0}[0,1]$. Indeed, if $\varphi(0) \neq 0$ and $\varphi(1) \neq 0$, then $C_{\varphi}\left(\mathcal{C}_{0}[0,1]\right)=\mathcal{C}[0,1]$; if $\varphi(0)=0$, then $C_{\varphi}\left(\mathcal{C}_{0}[0,1]\right)=\mathcal{C}_{0}[0,1]$; and finally if $\varphi(1)=0$, then $C_{\varphi}\left(\mathcal{C}_{0}[0,1]\right)=\{f \in \mathcal{C}[0,1]$ : $f(1)=0\}$.

Lemma 5.3. Let $\varphi$ be a continuous self-map of $[0,1]$. Assume that $V_{\varphi}$ acts on $L^{p}[0,1]$ with $1 \leqslant p<\infty$. If $\varphi$ is not strictly monotone, then $\overline{\operatorname{ran}}_{p} V_{\varphi}$ has infinite codimension. If $\varphi$ is strictly monotone, then $\overline{\operatorname{ran}}_{p} V_{\varphi}=L^{p}[0,1]$.

Proof. One can easily verify that $\overline{\operatorname{ran}}_{p} V_{\varphi} \cap \mathcal{C}[0,1]=\overline{\operatorname{ran}} V_{\varphi}$. Thus the result follows immediately from the previous lemma and the fact that both $\mathcal{C}_{0}[0,1]$ and $\{f \in \mathcal{C}[0,1]: f(1)=0\}$ are dense in $L^{p}[0,1]$.

The following lemma is an immediate consequence of Lemma 5.2.

Lemma 5.4. Let $\varphi$ be a continuous self-map of $[0,1]$ satisfying $\varphi(0)=0$. Assume that $V_{\varphi}$ acts on $\mathcal{C}_{0}[0,1]$. If $\varphi$ is not strictly increasing, then $\overline{\operatorname{ran}}_{0} V_{\varphi}$ has infinite codimension. If $\varphi$ is strictly increasing, then $\overline{\mathrm{ran}}_{0} V_{\varphi}=\mathcal{C}_{0}[0,1]$.

Now, we can show that the cyclicity of $V_{\varphi}$ is a severe restriction on $\varphi$.

Proposition 5.5. Let $\varphi$ be a continuous self-map of $[0,1]$. Assume that $V_{\varphi}$ acting on $L^{p}[0,1], 1 \leqslant p<\infty$, or on $\mathcal{C}[0,1]$ is cyclic. Then $\varphi$ is strictly monotone. In addition, if $\varphi(0)=0$ and $V_{\varphi}$ is cyclic when acting on $\mathcal{C}_{0}[0,1]$, then $\varphi$ is strictly increasing. 
Proof. It is known and easy to see that the closure of the range of a cyclic operator is at most of codimension 1. Thus it remains to apply Lemmas 5.2-5.4.

Since weakly supercyclic operators are cyclic, as a consequence of Propositions 5.1 and 5.5, we have the following corollary.

Corollary 5.6. Let $\varphi$ be a continuous self-map of $[0,1]$. If $V_{\varphi}$ acting on $L^{p}[0,1], 1 \leqslant p<\infty$, or on $\mathcal{C}_{0}[0,1]$ is weakly supercyclic, then $\varphi$ is strictly increasing and $\varphi(x) \leqslant x$ for $0 \leqslant x \leqslant 1$.

The cyclic properties that we shall be considering are cyclic, weakly supercyclic, weakly hypercyclic, supercyclic and hypercyclic. Actually, the real core of the question, whether a Volterra composition operator satisfies any of these properties or not, is in the friendly Hilbert space setting $L^{2}[0,1]$.

Proposition 5.7. Let $\varphi$ be a continuous self-map of $[0,1]$ with $\varphi(0)>0$ and $\varphi(1)>0$. Then $V_{\varphi}$ acting on $L^{2}[0,1]$ has a given cyclic property if and only if $V_{\varphi}$ acting on $L^{p}[0,1]$, $1 \leqslant p<\infty$, or on $\mathcal{C}[0,1]$ has the same cyclic property.

Proof. Let $1<p<\infty$. First, observe that $\mathcal{C}[0,1]$ is densely and continuously embedded into $L^{p}[0,1]$, and the latter space is densely and continuously embedded into $L^{1}[0,1]$. The same holds true if all the spaces carry their weak topologies. Thus it suffices to show that if $V_{\varphi}$ acting on $L^{1}[0,1]$ has a cyclic property, then so does $V_{\varphi}$ acting on $\mathcal{C}[0,1]$.

Suppose that $V_{\varphi}$ acting on $L^{1}[0,1]$ has a given cyclic property. By Proposition $5.5, \varphi$ is strictly monotone. By Lemma $5.2, V_{\varphi}$ acting on $\mathcal{C}[0,1]$ has dense range. Thus $V_{\varphi}$ is a bounded linear operator from $L^{1}[0,1]$ to $\mathcal{C}[0,1]$ with dense range. Therefore, we find that if $f$ in $L^{1}[0,1]$ provides a given cyclic property for $V_{\varphi}$ acting on $L^{1}[0,1]$, then $V_{\varphi} f$ provides the same property for $V_{\varphi}$ acting on $\mathcal{C}[0,1]$.

The proof of the next proposition is similar to the one of Proposition 5.7 and we omit it. One has to use Lemma 5.4 instead of Lemma 5.2.

Proposition 5.8. Let $\varphi$ be a continuous self-map of $[0,1]$ with $\varphi(0)=0$. Then $V_{\varphi}$ acting on $L^{2}[0,1]$ has a given cyclic property if and only if $V_{\varphi}$ acting on $L^{p}[0,1], 1 \leqslant p<\infty$, or on $\mathcal{C}_{0}[0,1]$ has the same cyclic property.

\subsection{Supercyclicity of $V_{\varphi}$ and hypercyclicity of $I+V_{\varphi}$ : case $\varphi(1)<1$}

Although $V_{\varphi}$ acting on $L^{2}[0,1]$ cannot be weakly hypercyclic, we have the following theorem.

THEOREM 5.9. Let $\varphi$ be a continuous strictly increasing self-map of $[0,1]$ such that $\varphi(x)<x$ for $0<x \leqslant 1$. Then $V_{\varphi}$ is supercyclic and $I+V_{\varphi}$ is hypercyclic.

Proof. Clearly, the sequence $\left\{\varphi_{n}(1)\right\}$ is strictly decreasing and tends to 0 as $n$ tends to $\infty$. One can easily verify that $\operatorname{ker} V_{\varphi}^{n}=\left\{f\right.$ such that $\left.\inf \operatorname{supp}(f) \geqslant \varphi_{n}(1)\right\}$. Now, a straightforward argument shows that $V_{\varphi}\left(\operatorname{ker} V_{\varphi}^{n+1}\right)$ is dense in $\operatorname{ker} V_{\varphi}^{n}$ for each positive integer $n$ and that $\operatorname{ker}^{\star} V_{\varphi}$ is dense in the underlying space. Therefore, the result follows from Proposition 4.12 and Corollary 4.3 . 
From Corollary 5.6, it follows that $\varphi$ cannot fail to be strictly increasing or to have the graph below the identity function. However, $\varphi(1)<1$ is a different issue.

\subsection{Supercyclicity of $V_{\varphi}$ : case $\varphi(1)=1$}

Although the Volterra operator is not weakly supercyclic (see [17]) there are supercyclic Volterra composition operators whose symbols are below the diagonal and take the value 1 at 1 . Along this subsection we will prove the following theorem.

THEOREM 5.10. Let $\varphi$ be a continuous strictly increasing self-map of $[0,1]$ with $\varphi(x)<x$ for $0<x<1$ and $\varphi(1)=1$ and analytic at 0 . If $\varphi^{\prime}(0)>\delta_{1}^{+}$, where

$$
\delta_{1}^{+}=\delta_{1}^{+}(\varphi)=\varlimsup_{x \rightarrow 1} \frac{1-x}{1-\varphi(x)},
$$

then $V_{\varphi}$ is supercyclic. In particular, if $\varphi$ is differentiable at 1 and $\varphi^{\prime}(0) \varphi^{\prime}(1)>1$, then $V_{\varphi}$ is supercyclic.

Proof. By Proposition 5.8, it is enough to show that $V_{\varphi}$ is supercyclic on $\mathcal{C}_{0}[0,1]$. We take $b>0$ with $1 / \varphi^{\prime}(0)<b<1 / \delta_{1}^{+}$and consider the dense subspace of $\mathcal{C}_{0}[0,1]$ defined by $E=\left\{f \in \mathcal{C}_{0}[0,1]: \inf \operatorname{supp}(f)>0\right\}$. According to Lemma 3.5, we have

$$
\varlimsup_{n \rightarrow \infty}\left\|V_{\varphi}^{n} f\right\|_{\infty}^{1 / n^{2}} \leqslant \sqrt{\delta_{1}^{+}} \text {for each } f \in E .
$$

On the other hand, by Theorem 3.6, $F=\left\{f \in V_{\varphi}^{\infty}\left(\mathcal{C}_{0}[0,1]\right): \varlimsup_{n \rightarrow \infty}\left\|V_{\varphi}^{-n} f\right\|_{\infty}^{1 / n^{2}} \leqslant \sqrt{b}\right\}$ is a dense linear subspace of $\mathcal{C}_{0}[0,1]$ satisfying $V_{\varphi}(F)=F=V_{\varphi}^{-1}(F)$. Let $S$ be the restriction of $V_{\varphi}^{-1}$ to $F$. Clearly, $V_{\varphi} S f=f$ for each $f$ in $F$ and

$$
\varlimsup_{n \rightarrow \infty}\left\|S^{n} f\right\|_{\infty}^{1 / n^{2}} \leqslant \sqrt{b} \text { for each } f \in F .
$$

Finally, take $b<c<1 / \delta_{1}^{+}$and let $\lambda_{n}=c^{n^{2} / 2}$ for $n \geqslant 0$. Inequalities (5.2) and (5.1) imply that $\lambda_{n} V_{\varphi}^{n} f$ tends to 0 as $n$ tends to $\infty$ for each $f$ in $E$ and $\lambda_{n}^{-1} S^{n} f$ tends to 0 for each $f$ in $F$. Upon applying Theorem 4.13 with $T=V_{\varphi}$ and $S_{k}=S^{k}$, we conclude that $V_{\varphi}$ acting on $\mathcal{C}_{0}[0,1]$ is supercyclic.

\subsection{Non-cyclicity}

The next theorem complements Theorem 5.10.

THEOREM 5.11. Let $\varphi$ be a continuous strictly increasing self-map of $[0,1]$ with $\varphi(x)<x$ for $0<x<1$ and $\varphi(1)=1$ and analytic at 1 . If $\varphi^{\prime}(1) \delta_{0}^{+}<1$, where

$$
\delta_{0}^{+}=\delta_{0}^{+}(\varphi)=\varlimsup_{x \rightarrow 0} \frac{\varphi(x)}{x}
$$

then $V_{\varphi}$ is not cyclic. In particular, if $\varphi$ is differentiable at 0 with $\varphi^{\prime}(0) \varphi^{\prime}(1)<1$, then $V_{\varphi}$ is not cyclic.

Proof. By Proposition 5.8, it is enough to prove that $V_{\varphi}$ is not cyclic on $L^{2}[0,1]$. Clearly, $\phi(x)=1-\varphi_{-1}(1-x)$ is continuous, strictly increasing, analytic at $0, \phi(x)<x$ for $0<x<1$, $\phi(1)=1, \phi^{\prime}(0)=1 / \varphi^{\prime}(1)$ and

$$
\delta_{1}^{+}(\phi)=\varlimsup_{x \rightarrow 1} \frac{1-x}{1-\phi(x)}=\delta_{0}^{+}(\varphi) .
$$


In addition, the fact that $\varphi^{\prime}(1) \delta_{0}^{+}(\varphi)<1$ implies $\phi^{\prime}(0)>\delta_{1}^{+}(\phi)$. Thus we may choose $1 \leqslant 1 / \phi^{\prime}(0)<b<1 / \delta_{1}^{+}(\phi)$. Since $\phi$ is analytic at zero, there is $0<a<1$ such that $\phi$ is analytic on $\left[0, \phi^{-1}(a)\right]$ and $\max _{\left[0, \phi^{-1}(a)\right]} 1 / \phi^{\prime} \leqslant b$. For each $n$ in $\mathbb{Z}$, we set $a_{n}=\phi_{-n}(a)$. We choose $a_{-1}<c<a_{0}$ and set $c_{n}=\phi_{-n}(c)$ for each $n$ in $\mathbb{Z}$. Clearly, $\left\{a_{n}\right\}$ and $\left\{c_{n}\right\}$ converge to 1 as $n$ tends to $+\infty$ and to 0 as $n$ tends to $-\infty$. Moreover, $c_{n}<a_{n}<c_{n+1}$ for each $n$ in $\mathbb{Z}$. By Lemma 3.8, there are non-zero functions $f_{0}$ in $\mathcal{F}\left[c_{0}, a_{0}\right]$ and $f_{1}$ in $\mathcal{F}\left[a_{-1}, c_{0}\right]$ that we extend to the whole interval $[0,1]$ by defining them as zero outside their intervals of definition. By Lemma 3.14, we find that $f_{0}$ as well as $f_{1}$ are in $V_{\phi}^{\infty}\left(\mathcal{C}_{0}[0,1]\right)$, which we defined in Subsection 8.3, and

$$
\varlimsup_{n \rightarrow \infty}\left\|V_{\phi}^{-n} f_{j}\right\|_{2}^{1 / n^{2}} \leqslant \sqrt{b} \quad \text { for } j=0,1 .
$$

On the other hand, Lemma 3.5 implies that

$$
\varlimsup_{n \rightarrow \infty}\left\|V_{\phi}^{n} f_{j}\right\|_{2}^{1 / n^{2}} \leqslant \sqrt{\delta_{1}^{+}(\phi)} \text { for } j=0,1 .
$$

Now, take real numbers $b<\alpha<\beta<1 / \delta_{1}^{+}(\phi)$ and set

$$
z_{n}= \begin{cases}\alpha^{n(1-n) / 2} & \text { if } n<0, \\ \beta^{n(n+1) / 2} & \text { if } n \geqslant 0 .\end{cases}
$$

From (5.3) and (5.4), it follows that $J(x \oplus y)=\sum_{n=-\infty}^{\infty} z_{n}\left(x_{n} V_{\phi}^{n} f_{0}+y_{n} V_{\phi}^{n} f_{1}\right)$ defines a bounded operator from $\ell^{2}(\mathbb{Z}) \oplus \ell^{2}(\mathbb{Z})$ into $L^{2}[0,1]$. We need to show that $J^{\star}$ has dense range. To this end, it is enough to check that $J$ is one-to-one. Let $x$ and $y$ be in $\ell^{2}(\mathbb{Z})$ and suppose that $J(x \oplus y)=0$. By Lemma 3.11, it follows that inf $\operatorname{supp}\left(V_{\phi}^{n} f_{0}\right)=c_{n}$ and $\inf \operatorname{supp}\left(V_{\phi}^{n} f_{1}\right)=a_{n-1}$ for each $n$ in $\mathbb{Z}$ and $\sup \operatorname{supp}\left(V_{\phi}^{n} f_{0}\right)=a_{n}$ and $\sup \operatorname{supp}\left(V_{\phi}^{n} f_{1}\right)=c_{n}$ for $n \leqslant 0$. Thus, for each $n \leqslant 0$, we find that $V_{\phi}^{n} f_{0}$ is different from zero and supported on $\left[c_{n}, a_{n}\right]$ and, for each $m \neq n$, we have that $V_{\phi}^{m} f_{j}$ vanishes on $\left[c_{n}, a_{n}\right]$. Similarly, for each $n \leqslant 0$, we find that $V_{\phi}^{n} f_{1}$ is different from zero and supported in $\left[a_{n-1}, c_{n}\right]$ and, for each $m \neq n$, we have that $V_{\phi}^{m} f_{j}$ vanishes on $\left[a_{n-1}, c_{n}\right]$. It follows that $x_{n}=y_{n}=0$ for $n \leqslant 0$. If $x \oplus y$ is different from zero, let $n$ be the minimal positive integer for which $\left|x_{n}\right|+\left|y_{n}\right|>0$. Since all $V_{\phi}^{m} f_{j}$ vanish on $\left[a_{n-1}, c_{n}\right]$, except for $m=n$ and $j=1$, it follows that $y_{n}=0$. Similarly, $x_{n}=0$, which is a contradiction. Therefore, $J$ is one-to-one.

Let $\left\{e_{n}\right\}_{n \in \mathbb{Z}}$ denote the canonical basis of $\ell^{2}(\mathbb{Z})$ and consider the (forward) weighted shift $S e_{n}=w_{n+1} e_{n+1}$, with weight sequence

$$
w_{n}=\frac{z_{n-1}}{z_{n}}= \begin{cases}\alpha^{n-1} & \text { for } n \leqslant 0, \\ \beta^{-n} & \text { for } n \geqslant 1 .\end{cases}
$$

We have $V_{\phi} J=J(S \oplus S)$. Therefore, $J^{\star} V_{\phi}^{\star}=\left(S^{\star} \oplus S^{\star}\right) J^{\star}$. In $[\mathbf{1 5}, \S 2]$, it is proved that $V_{\phi}^{\star}$ is unitarily similar under $(U f)(x)=f(1-x)$ to $V_{\varphi}$. Thus assuming that $V_{\varphi}$ is cyclic, then so is $V_{\phi}^{\star}$. Let $f$ in $L^{2}[0,1]$ be cyclic for $V_{\phi}^{\star}$. Then

$$
\operatorname{span}\left\{\left(S^{\star n} \oplus S^{\star n}\right)\left(J^{\star} f\right): n \geqslant 0\right\}=J^{\star}\left(\operatorname{span}\left\{V_{\phi}^{\star n} f: n \geqslant 0\right\}\right) .
$$

Since $J^{\star}$ has dense range, it follows that $J^{\star} f$ is cyclic for $S^{\star} \oplus S^{\star}$. Now, the operator $R$ on $\ell^{2}(\mathbb{Z})$, defined by $R e_{n}=(\alpha / \beta)^{|n(n+1)| / 2} e_{-n}, n$ in $\mathbb{Z}$, is bounded because $\alpha<\beta$. One easily checks that $S R=R S^{\star}$. Hence, $(I \oplus R)\left(S^{\star} \oplus S^{\star}\right)=\left(S^{\star} \oplus S\right)(I \oplus R)$. Therefore,

$$
\operatorname{span}\left\{\left(S^{\star} \oplus S\right)^{n}(I \oplus R)\left(J^{\star} f\right): n \geqslant 0\right\}=(I \oplus R)\left(\operatorname{span}\left\{\left(S^{\star} \oplus S^{\star}\right)^{n}\left(J^{\star} f\right): n \geqslant 0\right\}\right) .
$$

Taking into account that $J^{\star} f$ is cyclic for $S^{\star} \oplus S^{\star}$ and $I \oplus R$ has dense range, we see that $S^{\star} \oplus S$ is cyclic. Let $x \oplus y$ in $\ell^{2}(\mathbb{Z}) \oplus \ell^{2}(\mathbb{Z})$ be cyclic for $S^{\star} \oplus S$ and consider the dual pairing

$$
\langle u, v\rangle=\sum_{n \in \mathbb{Z}} u_{n} v_{n}, \quad u, v \in \ell^{2}(\mathbb{Z}) .
$$


Since $x \oplus y$ must be different from zero, the functional $\Phi(u \oplus v)=\langle u, y\rangle-\langle v, x\rangle$ on $\ell^{2}(\mathbb{Z}) \oplus$ $\ell^{2}(\mathbb{Z})$ is non-zero. However, for each $n \geqslant 0$, we have $\Phi\left(\left(S^{\star} \oplus S\right)^{n}(x \oplus y)\right)=\left\langle S^{\star n} x, y\right\rangle-$ $\left\langle S^{n} y, x\right\rangle=0$, which contradicts the fact that $x \oplus y$ is cyclic for $S^{\star} \oplus S$. The proof is complete.

\subsection{Hypercyclicity and supercyclicity of $V_{\varphi}$ on $\mathcal{C}_{0}[0,1)$}

Let $\mathcal{C}_{0}[0,1)$ be the Fréchet space of continuous functions vanishing at 0 endowed with the topology of uniform convergence on compact subsets of [0,1). Herzog and Weber [11] showed that $V_{\varphi}$, where $\varphi(x)=x^{b}$ with $0<b<1$, acting on $\mathcal{C}_{0}[0,1)$ is hypercyclic. Here, we shall provide an easy characterization in terms of the symbols $\varphi$ of the hypercyclicity of $V_{\varphi}$ acting on $\mathcal{C}_{0}[0,1)$. First, note that $V_{\varphi}$ acts from $\mathcal{C}_{0}[0,1)$ into itself if and only if $\varphi$ is a continuous map from $[0,1)$ into itself and $\varphi(0)=0$.

Proposition 5.12. Let $\varphi$ be a continuous self-map of $[0,1)$ with $\varphi(0)=0$.

(i) If $V_{\varphi}$ is weakly supercyclic on $\mathcal{C}_{0}[0,1)$, then $\varphi$ is strictly increasing.

(ii) If $\varphi$ is strictly increasing on $[0,1)$ and there are $0<a<b<1$ such that $\varphi(a)>a$ and $\varphi(b)=b$, then $V_{\varphi}$ is not weakly supercyclic on $\mathcal{C}_{0}[0,1)$.

(iii) If $\varphi$ is strictly increasing on $[0,1)$ and there is $0<b<1$ such with $\varphi(b)=b$, then $V_{\varphi}$ is not weakly hypercyclic on $\mathcal{C}_{0}[0,1)$.

Proof. To prove (i), observe that $\varphi(a)=\varphi(b)$ with $0<a<b<1$, then $\left(V_{\varphi} f\right)(a)=\left(V_{\varphi} f\right)(b)$ for each $f$ in $\mathcal{C}_{0}[0,1)$. Thus the range of $V_{\varphi}$ acting on $\mathcal{C}_{0}[0,1)$ is not dense and, therefore, $V_{\varphi}$ is not weakly supercyclic on $\mathcal{C}_{0}[0,1)$.

To prove (ii) and (iii), first observe that since $\varphi$ is increasing and $\varphi(b)=b$, we find that $\varphi$ is also a self-map of $[0, b]$. Let $P$ from $\mathcal{C}_{0}[0,1)$ onto $\mathcal{C}_{0}[0, b]$ be defined by $P f=\left.f\right|_{[0, b]}$. Set $\psi=\left.\varphi\right|_{[0, b]}$ and consider $V_{\psi}$ acting on $\mathcal{C}_{0}[0, b]$. Since $\varphi([0, b])=[0, b]$, we see that $P V_{\varphi}^{n} f=V_{\psi}^{n} P f$ for each $f$ in $\mathcal{C}_{0}[0,1)$. Thus weak hypercyclicity (weak supercyclicity) of $V_{\varphi}$ implies weak hypercyclicity (weak supercyclicity) of $V_{\psi}$. On the other hand, since $\left\|V_{\psi}\right\|<1$, we find that $V_{\psi}$ cannot be weakly hypercyclic on $\mathcal{C}_{0}[0, b]$ and, therefore, neither can $V_{\varphi}$ acting on $\mathcal{C}_{0}[0,1)$. Finally, assume that there is $0<a<b$ for which $\varphi(a)=\psi(a)>a$. Then the operator $V_{\psi}$ cannot be weakly supercyclic because of Corollary 5.6 and, therefore, neither can $V_{\varphi}$ acting on $\mathcal{C}_{0}[0,1)$.

We also need a lemma, which is a particular case of Theorem 3.2.5 in [23], dealing with projective limits of sequences of complete metrizable Abelian topological groups.

Lemma 5.13. Let $\left\{\mathcal{X}_{n}\right\}_{n \geqslant 0}$ be a sequence of $\mathcal{F}$-spaces and for each $m \geqslant n \geqslant 0$ let $T_{n, m}$ : $\mathcal{X}_{m} \rightarrow \mathcal{X}_{n}$ be a continuous operator with dense range satisfying that $T_{n, n}$ is the identity operator and $T_{k, n} T_{n, m}=T_{k, m}$ for $m \geqslant n \geqslant k$. Then $\bigcap_{n=0}^{\infty} T_{0, n}\left(\mathcal{X}_{n}\right)$ is dense in $\mathcal{X}_{0}$.

Theorem 5.14. Let $\varphi$ be a continuous self-map of $[0,1)$ with $\varphi(0)=0$. Then the following are equivalent.

(a) The operator $V_{\varphi}$ acting on $\mathcal{C}_{0}[0,1)$ is weakly hypercyclic.

(b) The operator $V_{\varphi}$ acting on $\mathcal{C}_{0}[0,1)$ is hypercyclic.

(c) The map $\varphi$ is strictly increasing and $\varphi(x)>x$ for $0<x<1$.

Proof. Clearly, (b) implies (a). According to Proposition 5.12, (a) implies (c). It remains to show that (c) implies (b). Thus, suppose that (c) is satisfied. Let $E$ be the space of bounded 
functions of $\mathcal{C}_{0}[0,1)$, which is clearly dense in $\mathcal{C}_{0}[0,1)$. Since $V_{\varphi}$ acting on $L^{\infty}[0,1]$ has norm strictly less than 1 , we see that $\left\|V_{\varphi}^{n} f\right\|_{\infty}$ tends to 0 as $n$ tends to $\infty$. Hence, $V_{\varphi}^{n} f$ tends to 0 in $\mathcal{C}_{0}[0,1)$ for each $f$ in $E$.

Since (c) implies that $\varphi(x)$ tends to 1 as $x$ tends to $1, \varphi$ extends to a one-to-one continuous map of $[0,1]$ onto itself. Take $0<a_{0}<1$ and set $a_{n}=\varphi_{n}\left(a_{0}\right)$ for each $n$ in $\mathbb{Z}$. Then $\left\{a_{n}\right\}$ tends to 1 as $n$ tends to $\infty$ and $\left\{a_{n}\right\}$ tends to 0 as $n$ tends to $-\infty$. Consider also the decreasing bilateral sequence of closed subspaces of $\mathcal{C}_{0}[0,1)$ defined by $G_{n}=\left\{f \in \mathcal{C}_{0}[0,1)\right.$ such that $\left.\left.f\right|_{\left[0, a_{n}\right]}=0\right\}, n \in \mathbb{Z}$. It is elementary to check that $V_{\varphi}\left(G_{n+1}\right)$ is a dense subspace of $G_{n}$ and $V_{\varphi}^{-1}\left(G_{n}\right)=G_{n+1}$ for each $n$ in $\mathbb{Z}$. Let $k$ be any integer. Applying Lemma 5.13 to $\mathcal{X}_{n}=G_{k+n}$ and $T_{n, m}: \mathcal{X}_{m} \rightarrow \mathcal{X}_{n}, m \geqslant n$, defined by the restriction of $V_{\varphi}^{m-n}$ to $\mathcal{X}_{m}=G_{k+m}$, we obtain $\bigcap_{n=0}^{\infty} V_{\varphi}^{n}\left(G_{k+n}\right)$ is dense in $G_{k}$ for each $k \in \mathbb{Z}$. Since $G=\bigcup_{n \in \mathbb{Z}} G_{k}$ is dense in $\mathcal{C}_{0}[0,1)$, it follows that $F=G \cap \bigcap_{n=0}^{\infty} V_{\varphi}^{n}\left(\mathcal{C}_{0}[0,1)\right)$ is dense in $\mathcal{C}_{0}[0,1)$.

Now, since $F$ is contained in the range of $V_{\varphi}$, for each $f$ in $F$ there is a unique $S f$ in $\mathcal{C}_{0}[0,1)$ such that $V_{\varphi} S f=f$. We also have $V_{\varphi}^{-1}(G)=G$ because $V_{\varphi}^{-1}\left(G_{n}\right)=G_{n+1}$ for each $n$ in $Z$ and, therefore, $V_{\varphi}^{-1}(F)=F$. Hence $S$ is well defined from $F$ into itself. Using that $V_{\varphi}^{-1}\left(G_{n}\right)=G_{n+1}$ once again, we see that inf supp $S^{n} f$ tends to 1 as $n$ tends to $\infty$ for each $f$ in $F$. Hence $S^{n} f$ tends to 0 as $n$ tends to $\infty$ for each $f$ in $F$. Applying Corollary 4.13 with $T_{n}=V_{\varphi}^{n}$ and $S_{n}=S^{n}$ and with $\lambda_{k}=1$, we obtain that $V_{\varphi}$ is hypercyclic.

Theorem 5.14 implies that $\varphi(x)<x$ for $0<x<1$ is not possible whenever $V_{\varphi}$ is hypercyclic on $\mathcal{C}_{0}[0,1)$. This is not true if we just consider supercyclicity.

Proposition 5.15. Let $\varphi$ be a strictly continuous self-map of $[0,1)$ with $\varphi(x)<x$ for $0<x<1$. Then $V_{\varphi}$ acting on $\mathcal{C}_{0}[0,1)$ is supercyclic.

Proof. Let $\left\{a_{n}\right\}$ be a strictly increasing sequence of positive numbers such that $\left\{a_{n}\right\}$ tends to 1 as $n$ tends to $\infty$ and let $P_{n}$ from $\mathcal{C}_{0}[0,1)$ onto $\mathcal{C}_{0}\left[0, a_{n}\right]$ be the projections defined by $P_{n} f=\left.f\right|_{\left[0, a_{n}\right]}$. Since $\varphi(x)<x$ for $0<x<1$, we obtain that $P_{n} V_{\varphi}^{k} f=V_{\psi_{n}}^{k} P_{n} f$ for each $f$ in $\mathcal{C}_{0}[0,1)$, where $\psi_{n}=\left.\varphi\right|_{\left[0, a_{n}\right]}$ and $V_{\psi_{n}}$ acts on $\mathcal{C}\left[0, a_{n}\right]$. By Theorem 5.9 the operators $V_{\beta_{n}}$, where $\beta_{n}(x)=a_{n}^{-1} \psi_{n}\left(a_{n} x\right)$, acting on $\mathcal{C}_{0}[0,1]$ are all supercyclic. Since a change of variables provides a similarity between $V_{\psi_{n}}$ acting on $\mathcal{C}_{0}\left[0, a_{n}\right]$ and $V_{\beta_{n}}$ acting on $\mathcal{C}_{0}[0,1]$, we see that each $V_{\psi_{n}}$ is also supercyclic.

For each $n \geqslant 0$, there is a dense $G_{\delta}$-set $M_{n}$ in $\mathcal{C}_{0}\left[0, a_{n}\right]$ such that each $f$ in $M_{n}$ is supercyclic for $V_{\psi_{n}}$. Since $P_{n}$ is continuous onto operator, we see that $W_{n}=P_{n}^{-1}\left(M_{n}\right)$ is a dense $G_{\delta}$-set in $\mathcal{C}_{0}[0,1)$. Thus, by Baire's theorem, $W=\bigcap_{n=0}^{\infty} W_{n}$ is a dense $G_{\delta}$-set in $\mathcal{C}_{0}[0,1)$. The fact that each $f$ in $W$ is supercyclic for $V_{\varphi}$ is straightforward. The proof is complete.

The only case not covered by Lemma 5.12, Theorem 5.14 and Proposition 5.15 is the one for which $\varphi$ is strictly increasing with $\varphi(x) \leqslant x$ for each $0 \leqslant x<1$ and there is $0<a<1$ for which $\varphi(a)=a$.

We close by showing a result that singles out the Volterra operator from a natural oneparametric family of Volterra composition operators.

Corollary 5.16. Assume that $\varphi_{\alpha}(x)=x^{\alpha}$ for $0 \leqslant x \leqslant \infty$, where $0<\alpha<1$. Then $V_{\varphi_{\alpha}}$ acting on $\mathcal{C}_{0}[0,1)$ is hypercyclic for $\alpha<1$, supercyclic for $\alpha>1$ and not weakly supercyclic for $\alpha=1$.

Proof. The statements for $\alpha<1$ and $\alpha>1$ follow from Propositions 5.15 and Theorem 5.14. It remains to show that the Volterra operator $V=V_{\varphi_{1}}$ is not weakly supercyclic on $\mathcal{C}_{0}[0,1)$. 
Suppose that it is weakly supercyclic. As in the proof of Proposition 5.12, we can see that $V$ must be weakly supercyclic on $\mathcal{C}_{0}[0, a]$ for each $0<a<1$, which is not the case, as shown in $[\mathbf{1 7}]$.

Acknowledgement. The authors would like to thank Patrick Ahern, David Drasin and the referee for very helpful comments and remarks.

\section{References}

1. J. B. Conway, A course in functional analysis, 2nd edn (Springer, New York, 1990).

2. I. Domanov, 'On the spectrum and eigenfunctions of the operator $(V f)(x)=\int_{0}^{x^{\alpha}} f(t) d t$ ', Perspectives in operator theory, Banach Center Publications 75 (Polish Academy of Sciences, Warsaw, 2007) 137-142.

3. I. Domanov, 'On the spectrum of the operator which is a composition of integration and substitution', Studia Math. 185 (2008) 49-65.

4. S. P. Eveson, 'Norms of iterates of Volterra operators on $L^{2}$ ', J. Operator Theory 50 (2003) 369-386.

5. S. P. Eveson, 'Asymptotic behaviour of iterates of Volterra operators on $L^{p}[0,1]$ ', Integral Equations and Operator Theory 53 (2005) 211-216.

6. E. A. Gallardo-Gutiérrez and A. Montes-Rodríguez, 'The Volterra operator is not supercyclic', Integral Equations Operator Theory 50 (2004) 211-216.

7. M. González, F. León-Sandedra and A. Montes-Rodríguez, 'Semi-Fredholm theory: hypercyclic and supercyclic subspaces', Proc. London Math. Soc. 81 (2000) 169-189.

8. S. GRIVAux, 'Hypercyclic operators, mixing operators and the bounded steps problem', J. Operator Theory 54 (2005) $147-168$.

9. K. Grosse-Erdmann, 'Universal families and hypercyclic operators', Bull. Amer. Math. Soc. 36 (1999) $345-381$.

10. D. A. Herrero, 'Limits of hypercyclic and supercyclic operators', J. Funct. Anal. 99 (1991) 179-190.

11. G. Herzog and A. Weber, 'A class of hypercyclic Volterra type operators', Demonstratio Math. 39 (2006) $465-468$.

12. Y. KatZnelson, An introduction to harmonic analysis (Dover, New York, 1976).

13. F. León-SaAvedra and A. Montes Rodríguez, 'Linear structure of hypercyclic vectors', J. Funct. Anal. 148 (1997) 524-545.

14. B. Ja. Levin, Distribution of zeros of entire functions (American Mathematical Society, Providence, RI, 1980).

15. A. Montes-Rodríguez, A. Rodríguez-Martínez and S. Shkarin, 'Spectral theory of Volterra composition operators', Math. Z. 261 (2009) 431-472.

16. A. Montes-Rodríguez and H. Salas, 'Supercyclic subspaces: spectral theory and weighted shifts', Adv. Math. 163 (2001) 74-134.

17. A. Montes-Rodríguez And S. A. Shkarin, 'Non-weakly supercyclic operators', J. Operator Theory 58 (2007) 39-62.

18. W. Rudin, Real and complex analysis, McGraw-Hill, New York, 1971.

19. H. N. Salas, 'Hypercyclic weighted shifts', Trans. Amer. Math. Soc. 347 (1995) 993-1004.

20. H. N. Salas, 'Supercyclicity and weighted shifts', Studia Math. 135 (1999) 55-74.

21. L. Schwarz, Analyse mathematique, Hermann, Paris, 1967.

22. Y. S. Tong, 'Quasinilpotent integral operators', Acta Math. Sinica 32 (1989) 727-735.

23. J. Wengenroth, Derived functors in functional analysis, Lecture Notes in Mathematics 1810 (Springer, Berlin, 2003).

24. R. Whitley, 'The spectrum of a Volterra composition operator', Integral Equations Operator Theory 10 (1987) 146-149.

\author{
Alfonso Montes-Rodríguez and Alejandro \\ Rodríguez-Martínez \\ Departamento de Análisis Matemático \\ Facultad de Matemáticas \\ Universidad de Sevilla, Aptdo. 1160 \\ Sevilla 41080 \\ Spain
}

arm@us.es

\author{
Stanislav Shkarin \\ Department of Pure Mathematics \\ Queen's University Belfast \\ University road \\ Belfast \\ BT7 1NN \\ United Kingdom \\ s.shkarin@qub.ac.uk
}

